Massachusetts Institute of Technology

Department of Economics

Working Paper Series

\title{
AGGREGATE COMPARATIVE STATICS
}

\author{
Daron Acemoglu \\ Martin Kaae Jensen
}

Working Paper 09-09

February 22, 2009

Room E52-251

50 Memorial Drive

Cambridge, MA 02142

This paper can be downloaded without charge from the Social Science Research Network Paper Collection at http://ssrn.com/abstract $=1374641$ 


\section{Digitized by the Internet Archive in 2011 with funding from Boston Library Consortium Member Libraries}




\title{
Aggregate Comparative Statics*
}

\author{
Daron Acemoglu ${ }^{\dagger}$ and Martin Kaae Jensen ${ }^{\ddagger}$
}

February 22, 2009

\begin{abstract}
In aggregative games, each player's payoff depends on her own actions and an aggregate of the actions of all the players (for example, sum, product or some moment of the distribution of actions). Many common games in industrial organization, political economy, public economics, and macroeconomics can be cast as aggregative games. In most of these situations, the behavior of the aggregate is of interest both directly and also indirectly because the comparative statics of the actions of each player can be obtained as a function of the aggregate. In this paper, we provide a general and tractable framework for comparative static results in aggregative games. We focus on two classes of aggregative games: (1) aggregative of games with strategic substitutes and (2) "nice" aggregative ganes, where payoff functions are continuous and concave in own strategies. We provide simple sufficient conditions under which "positive shocks" to individual players increase their own actions and have monotone effects on the aggregate. We show how this framework can be applied to a variety of examples and how this enables more general and stronger comparative static results than typically obtained in the literature.
\end{abstract}

"We would like to thank Roger Hartley, Jean-Francois Mertens, Alex Possajennikov, and Burkhard Schipper for their helpful remarks and suggestions. Thanks also to seminar participants at the University of Copenhagen, University of Manchester, and at the 2008 Midlands Game Theory Workshop in Birmingham. All remaining errors are of course our responsibility.

${ }^{\dagger}$ Department of Economics, Massachusetts Institute of Technology (e-mail: daron@mit.edu)

${ }^{\ddagger}$ Department of Economics, University of Birmingham. (e-mail: m.k.jensen@bham.ac.uk) 


\section{Introduction}

In aggregative games, each player's payoff depends on her own actions and some aggregate of all players' actions. For example, the Cournot model of oligopoly competition is an aggregative game; each firm's profits depend on its own quantity and total quantity supplied to the market. More generally, the aggregate could be any mapping from the players' action profile to a real number. ${ }^{1}$. Numerous games studied in the literature can be cast as aggregative games. These include the majority of the models of competition (Cournot and Bertrand with or without product differentiation), models of

(patent) races, models of contests and fighting, models of public good provision, models with aggregate demand externalities, and so on. In many applied problems, the focus is naturally on how the aggregate (e.g., total supply to the market, the price index, probability of innovation, total public good provision) responds to changes in the environment. In addition, comparative statics of individual actions can often be obtained as a function of the aggregate. ${ }^{2}$

In this paper, we provide a simple general framework for comparative static analysis in aggregative games. Our approach is applicable to diverse environments that can be cast as aggregative games and enables us to provide sufficient conditions for a rich set of comparative static results. We present two sets of results. First, we focus on aggregative games with strategic substitutes. In games with strategic substitutes, each player's payoff function is supermodular in her own strategy and exhibits decreasing differences in her own strategy and the strategy vector of other players. For aggregative games with strategic substitutes, we establish the following results:

1. Changes in parameters that only affect the aggregate (such as a shift in demand in the Cournot game) always lead to an increase in the aggregate - in the sense that the smallest and the largest elements of the set of equilibrium aggregates increase.

2. Entry of an additional player decreases the (appropriately-defined) aggregate of the existing players.

3. A "positive" idiosyncratic shock, defined as a parameter change that increases the marginal payoff 'of a single player, leads to an increase in that player's strategy and a decrease in the other players' aggregate.

\footnotetext{
${ }^{1}$ We discuss games with multi-dimensional aggregates in Section 7.

${ }^{2}$ The fact that a game is aggregative does not imply that players ignore the impact of their strategies on aggregates. When they do so, we say that the equilibrium is Walrasian Nash or that the play is Walrasian. Our results are generalized to Walrasian Nash equilibria in Section 9. Because in this case there are more more limited "game-theoretic" interactions, the analysis is more straightforward.
} 
The comparative static results mentioned above are intuitive. But it is surprising that for aggregative games, they hold at the same level of generality as the monotonicity results for supermodular games (in particular, no quasi-concavity or convexity assumptions are needed).

Nevertheless, not all aggregative games exhibit strategic substitutes. The second set of results we present focus on "nice" aggregative games, which are games with payoff functions that are continuous, concave (or pseudo-concave) in own strategies, and sufficiently smooth (in particular, twice continuously differentiable). Many games analyzed in industrial organization, political economy and other areas satisfy these conditions. An example is contest (or fighting) games, where payoffs are determined by the effort levels of all players; contests typically exhibit neither strategic substitutes nor complements. Another example is provided by games in which players make voluntary contributions to the provision of a public good.

We prove parallel results for nice aggregative games under an additional technical assumption, which we refer to as local solvability. Under this assumption, which ensures the local invertibility of the "backward reply" mapping described further below, we establish a general monotonicity theorem similar in spirit to the monotonicity results for supermodular games. This theorem implies that a positive shock to (one or more of) the players leads to an increase in the smallest and largest equilibrium values of the aggregate. We also prove that entry of an additional player increases the aggregate and derive more extensive "individual comparative static" results.

An informal summary of our results from both aggregative games with strategic substitutes and from nice aggregative games is that, under a variety of reasonable economic conditions, comparative statics are "regular" (for example, a reduction marginal cost increases output and so on). Nevertheless, it should be emphasized that there is no guarantee that such intuitive results should hold generally. The next example illustrates how counterintuitive, "perverse," results can arise in simple games, even in simple aggregative games.

Example 1 Consider three players $i=1,2,3$ with payoff functions $\pi_{i}(s)=-0.5 s_{i}^{2}+\alpha_{i}(1-$ $\left.\alpha_{i}\right)^{-1}\left(\sum_{j \neq i} s_{j}\right) s_{i}+\beta_{\imath}\left(1-\alpha_{i}\right)^{-1} s_{i}$ defined locally in a sufficiently large neighborhood of the equilibrium found below. Assume that $\alpha_{1}>1,1>\alpha_{2}>0, \alpha_{3}<0, \beta_{1}<0, \beta_{2}>0, \alpha_{1}+\alpha_{2}+\alpha_{3}>1$, $\beta_{1}+\beta_{2}+\beta_{3}<0$, and $\alpha_{1}+\alpha_{3}<1$.

This is an aggregative game since we can write the payoffs as a function of players' own strategies and the aggregate $Q=\sum_{j} s_{j}: \Pi_{i}\left(s_{i}, Q\right)=-0.5 s_{i}^{2}+\alpha_{i}\left(1-\alpha_{i}\right)^{-1}\left(Q-s_{i}\right) s_{i}+\beta_{i}\left(1-\alpha_{i}\right)^{-1} s_{i}$. By strict concavity, best response functions in this game are: $r_{i}\left(s_{-i}\right)=\alpha_{i}\left(1-\alpha_{i}\right)^{-1}\left(\sum_{j \neq i} s_{j}\right)+$ $\beta_{i}\left(1-\alpha_{i}\right)^{-1}$. Solving for the pure strategy Nash equilibrium $\left(s_{i}^{*}=r_{i}\left(s_{-i}^{*}\right), i=1,2,3\right)$ we obtain $s_{i}^{*}=\alpha_{i} Q^{*}+\beta_{i}$, where $Q^{*}=s_{1}^{*}+s_{2}^{*}+s_{3}^{*}=\left(\beta_{1}+\beta_{2}+\beta_{3}\right)^{-1}\left(1-\alpha_{1}-\alpha_{2}-\alpha_{3}\right)$ is the equilibrium aggregate. Now consider a (small) increase in $\alpha_{2}$. This is a "positive shock" to player 2: holding 
opponents' strategies fixed, it increases player 2's marginal payoff and therefore "lifts" player 2 's best response function, ${ }^{3} \partial r_{2}\left(s_{-2}\right) / \partial \alpha_{2}>0$. yet But this positive direct effect on player 2 's optimal strategy notwithstanding, an increase in $a_{2}$ leads to a decrease in player 2 's strategy in equilibrium:

$$
\frac{\partial s_{i}^{*}}{\partial \alpha_{2}}=Q^{*}+\alpha_{2} \frac{\partial Q^{*}}{\partial \alpha_{2}}=\frac{\beta_{1}+\beta_{2}+\beta_{3}}{1-\alpha_{1}-\alpha_{2}-\alpha_{3}}+\alpha_{2} \frac{\beta_{1}+\beta_{2}+\beta_{3}}{\left(1-\alpha_{1}-\alpha_{2}-\alpha_{3}\right)^{2}}<0
$$

As can also be seen, the positive shock to player 2 leads to a decrease in the equilibrium aggregate:

$$
\frac{\partial Q^{*}}{\partial \alpha_{2}}<0 \text {. }
$$

In summary, a parameter change that unambiguously increases the marginal payoff for a player, which should, all else equal, lead to an increase in that player's strategy and the aggregate, in fact leads to a decrease in the player's strategy in equilibrium as well as a decrease in the aggregate.

Part of the contribution of our paper is to provide minimal conditions to ensure that "perverse" results, such as those described in the previous example, do not apply. In particular, such results are ruled out in nice aggregative games by the local solvability assumption mentioned above. In addition to providing minimal conditions for general comparative static results and significantly weakening the conditions that are available in the literature (for example, for models of patent races, contests, and public good provision), our approach is also useful because it highlights the common features that ensure "regular" comparative static results. These results are made possible by the alternative approach we use for deriving comparative static results (the more familiar approaches in the literature rely on the implicit function theorem and lattice-theoretic tools in the case of supermodular games).

Our approach can be explained as follows. Consider a general comparative static problem written as

$$
\mathbf{A} \cdot \mathbf{d s}=-\mathbf{b} \cdot d t
$$

where $d t \in \mathbb{R}$ is the change in some exogenous variable, ds $\in \mathbb{R}^{M}$ designates the induced change in the endogenous variables, $\mathbf{A}$ is an $M \times M$ matrix and $\mathbf{b}$ is an $M$-dimensional vector. An important question is to specify the conditions under which we can sign ds "robustly"-meaning without specifying numerical values for the elements of the matrix $\mathbf{A}$ and vector $\mathbf{b}$. Cast in this generality, the conclusion is somewhat depressing: to obtain such results it is necessary to ascertain the sign of the elements of $A^{-1}$. But even when $A$ is symmetric negative definite, we

\footnotetext{
${ }^{3}$ In other words, player 2's payoff function exhibits increasing differences in $s_{2}$ and $\alpha_{2}$ (Topkis (1978)). This is an equivalent way of defining a "positive shock" when strategy sets are one-dimensional and payoff functions are concave.
} 
can do this only when one of the following two additional (and stringent) conditions hold: (i) when $\mathbf{A}$ is a Metzler matrix, that is, it has non-negative off-diagonal elements, or (ii) when $\mathbf{A}$ is a Morishima matrix, that is, it becomes a Metzler matrix when we reverse the sign of one or more variables. ${ }^{4}$ The only general case where these conditions are satisfied is provided by supermodular games. Since many games that arise in applications are not supermodular, much of the applied literature imposes additional parametric restrictions in the context of specific games to derive comparative statics results. The discussion above highlights that many of these conclusions may not be robust and in general there are no systematic investigations of when the specific conclusions enjoy such robustness.

Our alternative approach is not to impose parametric restrictions, but to exploit the aggregative nature of the games in question and note that what is often of economic interest is not the entire vector $\mathrm{ds}$, but the behavior of the appropriate aggregate, or just one of its coordinates (the latter corresponds to deriving robust results for a single player as opposed to all players). With this perspective, robust and general comparative static results can be obtained under considerably weaker conditions. Our contribution is to suggest this perspective and show how it can be made operational.

Our paper is related to a number of different strands in the literature. Comparative static results in most games are obtained using the implicit function theorem. The main exception is for supermodular games (games with strategic complements). Topkis (1978, 1979), Milgrom and Roberts (1990) and Vives (1990) provide a framework for deriving comparative static results in such games. These methods do not extend beyond supermodular games.

More closely related to our work, and in many ways its precursor, is Corchón (1994). Corchón (1994) provides comparative static results for aggregative games with strategic substitutes, but only under fairly restrictive conditions, which, among other things, ensure uniqueness of equilibria. These conditions are typically not easy to check in many applied problems. In contrast, we provide general comparative static results for aggregative games with strategic substitutes without imposing any additional assumptions. We also provide parallel but stronger results for nice aggregative games without strategic substitutes. Another similarity between our paper and Corchón (1994) is that both make use of the so-called backward reply correspondence of Selten (1970). In an aggregative game, the backward reply correspondence gives the (best response) strategies of players that are compatible with a given value of the aggregate. ${ }^{5}$ In a seminal paper,

\footnotetext{
${ }^{4}$ See Bassett et al (1968) and Hale et al (1999).

${ }^{5}$ The first systematic study of aggregative games (German: aggregierbaren Spiele) can be found in Selten (1970). After defining aggregative games, Selten proceeds to define what he calls the Einpassungsfunktion (Selten (1970), p. 154), that is, the backward reply function of an individual player. As Selten proves, the backward reply correspondence is single-valued (a function) provided that the player's best-response function has slope greater
} 
Novshek (1985) used this correspondence to give the first general proof of the existence of purestrategy equilibria in the Cournot model without assuming quasi-concavity of payoff functions (see also Kukushkin (1994)). Novshek's result has since been strengthened and generalized to a larger class of aggregative games (e.g., Dubey et al. (2006) and Jensen (2007)) and our results on games with strategic substitutes utilize Novshek (1985)'s construction in the proofs. ${ }^{6}$ Our results on "nice" aggregative games blend the backward reply approach with the equilibrium comparison results reported in Milgrom and Roberts (1994) and Villas-Boas (1997). ${ }^{7}$

The rest of the paper is organized as follows. Section 2 provides basic definitions. Section 3 presents a number of common examples, which can be cast as aggregative games. Section 4 provides the general comparative static results for aggregative games with strategic substitutes. Section 5 generalizes and strengthens these results for "nice" aggregative games, which feature payoffs that are continuous and (pseudo-)concave in own strategies. Section 6 shows how the results from Sections 4 and 5 can be used to obtain general characterization results in various applications. Section 7 discusses how these results can be extended to games with multi-dimensional aggregates and Section 8 provides additional generalizations of the results presented in Section 5 . Section 9 briefly discusses Walrasian Nash equilibria (cf. footnote 2). Section 10 concludes and the Appendix contains the proofs omitted from the text.

\section{Basic Definitions}

In this section, we introduce some basic definitions.

Let $\Gamma=\left(\pi_{i}, S_{i}, T\right)_{i \in \mathcal{I}}$ denote a noncooperative game with a finite set of players $\mathcal{I}=\{1, \ldots, I\}$, and finite-dimensional strategy sets $S_{i} \subseteq \mathbb{R}^{N}$. In addition, $T \subseteq \mathbb{R}^{M}$ is a set of exogenous parameters with typical element $t \in T$. We will focus on how the set of equilibria of $\Gamma$ changes in response to changes in $t$.

Throughout the rest of the paper we assume that the joint strategy set

$$
S \equiv \prod_{i \in \mathcal{I}} S_{i}
$$

than -1. The assumptions imposed by Corchón (1994) imply that the slope of players' best-response functions lie strictly between -1 and 0 , so that the backward reply correspondence is both single-valued and decreasing. Neither is necessarily the case in many common games and neither is imposed in this paper.

${ }^{6}$ Novshek's explicit characterization of equilibria is similar to the characterization of equilibrium in supermodular games that uses the fixed point theorem of Tarski (1955). Both of these enable the explicit study of the behavior of "largest" and "smallest" fixed points in response to parameter changes. Tarski's result is used, for example, in the proof of Theorem 6 in Milgrom and Roberts (1990).

${ }^{7}$ More specifically, our proofs repeatedly use that the smallest and largest fixed points of a continuous function from a subset of real numbers into itself will increase when the curve is "shifted up" (see Figure 1 of Villas-Boas (1997) or Figure 2 of Milgrom and Roberts (1994)). 
is compact (in the usual topology) and the payoff functions

$$
\pi_{i}: S \times T \rightarrow \mathbb{R}
$$

are upper semi-continuous for each $i \in \mathcal{I}$. Let

$$
R_{i}\left(s_{-i}, t\right) \equiv \arg \max _{s_{i} \in S_{i}} \pi_{i}\left(s_{i}, s_{-i}, t\right)
$$

denote the best response correspondence (with the standard notation $s_{-i} \in S_{-i} \equiv \prod_{j \neq i} S_{j}$ ). Given the compactness and upper semi-continuity assumptions, the correspondence $R_{i}$ is non-emptyand compact-valued, and upper hemi-continuous.

We next define the notion of an aggregator.

Definition 1 (Aggregator) A mapping $g: S \rightarrow \mathbb{R}^{K}$ (with $K \leq N$ ) is an aggregator if it is continuous, increasing and separable across the players, i.e., if there exists a strictly increasing function $H: \mathbb{R}^{K} \rightarrow \mathbb{R}^{K}$ and increasing functions $h_{i}: S_{i} \rightarrow \mathbb{R}^{K}$ (for each $i \in \mathcal{I}$ ) such that:

$$
g(s)=H\left(\sum_{j=1}^{I} h_{j}\left(s_{j}\right)\right) .
$$

Throughout this paper $K$ is referred to as the dimension of the aggregate. For most of the analysis (in particular, until Section 7 ), we impose $K=1$, but throughout there are no restrictions on $N$. In particular, except Corollary 3 in Section 8 , none of our results require $N=1$ (onedimensional strategy sets). The requirement that $g$ is increasing in $s$ ensures that both $g$ and $-g$ cannot be aggregators for the same game. Naturally, since we can change the order on individual strategies (thus working with $-s_{i}$ instead of $s_{i}$ for some $i$ ), this requirement is not very restrictive. Common examples, such as the sum of strategies $g(s)=\sum_{j=1}^{I} s_{j}$, satisfy the definition (with $h_{i}\left(s_{i}\right)=s_{i}$ and $H(z)=z$ ). Two other simple examples are $g(s)=\left(\alpha_{1} s_{1}^{\beta}+\ldots+\alpha_{N} s_{N}^{\beta}\right)^{1 / \beta}$, $S \subseteq \mathbb{R}^{N}$, and $g(s)=\prod_{j=1}^{I} s_{j}^{\alpha_{3}}, S \subseteq \mathbb{R}_{++}^{N}$ where $\alpha_{j}>0$ (for each $j$ ) and $\beta>0$, which are, respectively, a CES function and a Cobb-Douglas function. ${ }^{8}$

Remark 1 (Differentiability and Aggregation) In the case of one-dimensional strategy sets, Definition 1 is the standard definition of separability when $g$ is strictly increasing (see, e.g., Gorman (1968)). It can be easily established that when $g$ is twice continuously differentiable, $N=K=1$, and $I \geq 3$, it is separable if and only if the "marginal rate of transformation" between any two players $i$ and $j$ is independent of the other players' actions; that is,

$$
\frac{D_{s_{2}} g(s)}{D_{s_{j}} g(s)}=h_{i, j}\left(s_{i}, s_{j}\right) \text { for all } s \in S
$$

\footnotetext{
${ }^{8}$ In the first case $h_{2}\left(s_{i}\right)=\alpha_{i} s_{2}^{\beta}$ (with $\left.s_{i}>0\right)$ and $H(z)=z^{1 / \beta}$. In the second $h_{i}\left(s_{i}\right)=\alpha_{i} \log \left(s_{z}\right)$ and $H(z)=$ $\exp (z)$ (again with $s_{i}>0$ ).
} 
where $h_{i, j}: S_{i} \times S_{j} \rightarrow R$ is a function of $s_{i}$ and $s_{j}$, but not of any $s_{q}$ with $q \neq i, j$. More generally, when $g$ is twice continuously differentiable, strictly increasing, and $I \geq 3$, it may be verified that it satisfies Definition 3 if and only if there exist increasing functions $f_{i}: S_{i} \times \mathbb{R}^{K} \rightarrow \mathbb{R}^{N}$ such that for each player $i \in \mathcal{I}$ :

$$
D_{s_{i}} g(s)=f_{i}\left(s_{i}, g(s)\right) \text { for all } s \in S \text {. }
$$

When $g$ is increasing (and not necessarily strictly increasing), as is the case in Definition 1 , (3) is still implied provided that $g$ is also twice continuously differentiable. This observation will play an important role in Section 5. Clearly, equation (3) also gives an alternative and often very convenient way of verifying that a strictly increasing function $g$ is an aggregator.

Definition 2 (Aggregative Game) The game $\Gamma=\left(\pi_{i}, S_{i}, T\right)_{i \in \mathcal{I}}$ is aggregative if there exists an aggregator $g: S \rightarrow \mathbb{R}^{K}$ and a reduced payoff function

$$
\Pi_{i}: S_{i} \times \mathbb{R}^{K} \times T \rightarrow \mathbb{R}
$$

for each player $i$ such that

$$
\Pi_{i}\left(s_{i}, g(s), t\right) \equiv \pi_{i}\left(s_{i}, s_{-i}, t\right) .
$$

Clearly, an aggregative game is fully summarized by the tuple $\left(\left(\Pi_{2}, S_{2}\right)_{i \in \mathcal{I}}, g, T\right)$.

Another key concept we will use is games with strategic substitutes. ${ }^{9}$ We say that the payoff function $\pi_{i}\left(s_{i}, s_{-i}, t\right)$ exhibits decreasing differences in $s_{i}$ and $s_{-i}$ if for all $s_{i}^{\prime}>s_{i}$, the "difference" $\pi_{i}\left(s_{i}^{\prime}, s_{-i}, t\right)-\pi_{i}\left(s_{i}, s_{-i}, t\right)$ is a decreasing function in each of the coordinates of $s_{-i} \in S_{-i} \subseteq$ $\mathbb{R}^{N(I-1)}$. When $\pi_{i}$ is twice differentiable, this will hold if and only if $D_{s_{2} s_{3}}^{2} \pi_{i}\left(s_{i}, s_{-i}, t\right)$ is a nonpositive matrix for all $j \neq i$. The payoff function $\pi_{i}\left(s_{i}, s_{-i}, t\right)$ is supermodular in $s_{i}$ if $\pi_{i}\left(s_{i} \vee\right.$ $\left.\tilde{s}_{i}, s_{-i}, t\right)+\pi_{i}\left(s_{i} \wedge \tilde{s}_{i}, s_{-i}, t\right) \geq \pi_{i}\left(s_{i}, s_{-i}, t\right)+\pi_{i}\left(\tilde{s}_{i}, s_{-i}, t\right)$ for all $s_{i}, \tilde{s}_{i} \in S_{i}$ (and $s_{-i} \in S_{-i}, t \in T$ ). Here $s_{i} \vee \tilde{s}_{i}\left(s_{i} \wedge \tilde{s}_{i}\right)$ denotes the coordinatewise maximum (minimum) of the vectors $s_{i}$ and $\tilde{s}_{i}$. Naturally, this definition requires that $s_{i} \vee \tilde{s}_{i}$ and $s_{i} \wedge \tilde{s}_{i}$ are contained in $S_{i}$ whenever $s_{i}, \tilde{s}_{i} \in S_{i}$, i.e., $S_{i}$ must be a lattice. When strategy sets are one-dimensional, supermodularity as well as the lattice-structure of strategy sets are automatically satisfied, so only decreasing differences remains to be checked. For multi-dimensional strategy sets, supermodularity holds for twice differentiable payoff functions if and only if $D_{s_{i}^{n} s_{i}^{m}}^{2} \pi_{i}\left(s_{i}, s_{-i}, t\right) \geq 0$ for all $m \neq n$ (where $s_{i}^{n}$ and $s_{i}^{m}$ denote the $n$th and $m$ th components of of the strategy vector $s_{\imath}$ of player $i$ ).

\footnotetext{
${ }^{9}$ When a game does not feature strategic substitutes, we will impose additional conditions, and in particular, we will focus on "nice games" that satisfy certain concavity and differentiability conditions. Since we do not make use of these until Section 5, we do not introduce this concept until then.
} 
Definition 3 (Strategic Substitutes) The game $\Gamma=\left(\pi_{i}, S_{i}\right)_{i \in \mathcal{I}}$ is a game with strategic substitutes if strategy sets are lattices and each player's payoff function $\pi_{i}\left(s_{i}, s_{-i}, t\right)$ is supermodular in $s_{i}$ and exhibits decreasing differences in $s_{i}$ and $s_{-i}$.

Equivalently, we will also say that a game has (or features) strategic substitutes. A game that is both aggregative and has strategic substitutes, is an aggregative game with strategic substitutes. Notice that decreasing differences usually is straightforward to verify in aggregative games. In fact, when the aggregator $g$ is a symmetric function there will only be one condition to check for each player. For instance, consider an aggregative game with linear aggregator $g(s)=\sum_{j=1}^{I} s_{j}$ and one-dimensional strategy sets, so that $\Pi_{i}\left(s_{i}, \sum_{j=1}^{I} s_{j}, t\right) \equiv \pi_{i}\left(s_{i}, s_{-i}, t\right)$. If $\pi_{i}$ is sufficiently smooth, then decreasing differences is equivalent to nonpositive cross-partials, i.e., $D_{s_{i} s_{j}}^{2} \pi_{i}=$ $D_{12}^{2} \Pi_{i}+D_{22}^{2} \Pi_{i} \leq 0$. This immediately implies that if decreasing differences holds for some opponent $j$, it must hold for all opponents.

Remark 2 (Strategic Substitutes and Decreasing Differences in s and Q) Unless players take $Q$ as given (as in Walrasian Nash equilibria in Section 9), there is no tight relationship between strategic substitutes and the condition that $\Pi_{i}\left(s_{i}, Q\right)$ exhibits decreasing differences in $s_{i}$ and $Q$ (the latter may be thought of as "stratcgic substitutes in $s_{i}$ and the aggregate $Q$ "). For example, suppose that $N=1, g(s)=\sum_{q=1}^{I} s_{i}$, and assume that payoff functions are twice differentiable. Then strategic substitutes requires that $D_{s_{i} s_{j}}^{2} \Pi_{i}\left(s_{i}, \sum_{q=1}^{I} s_{q}\right)=D_{12}^{2} \Pi_{i}\left(s_{i}, Q\right)+D_{22}^{2} \Pi_{i}\left(s_{i}, Q\right) \leq 0$ where $Q=\sum_{q=1}^{I} s_{q}$. Decreasing differences in $s_{i}$ and $Q$ requires that $D_{12}^{2} \Pi_{i}\left(s_{i}, Q\right) \leq 0$. Clearly neither condition implies the other. If $D_{22}^{2} \Pi_{i}\left(s_{i}, Q\right) \leq 0$, then our strategic substitutes condition is weaker and we can have $D_{12}^{2} \Pi_{i}\left(s_{i}, Q\right)>0$ in a game with strategic substitutes.

Finally, we define an equilibrium in the standard fashion.

Definition 4 (Equilibrium) Let $\left(\left(\Pi_{i}, S_{i}\right)_{i \in \mathcal{I}}, g, T\right)$ be an aggregative game. Then $s^{*}(t)=$ $\left(s_{1}^{*}(t), \ldots, s_{I}^{*}(t)\right)$ is a (pure-strategy Nash) equilibrium if for each player $i \in \mathcal{I}$,

$$
s_{i}^{*}(t) \in \arg \max _{s_{i} \in S_{2}} \Pi_{i}\left(s_{i}, g\left(s_{i}, s_{-i}^{*}\right), t\right)
$$

\section{Examples of Aggregative Games}

In this section, we present several games that have been widely studied in the literature. In each case, we show how they can be cast as aggregative games. For now, we focus on games with onedimensional aggregates, returning to the those with multi-dimensional aggregates in Section 7 . Throughout this section, we drop dependence on the parameter vector $t \in T$ to simplify notation. 


\subsection{Cournot Competition}

As already mentioned in the Introduction, Cournot games are aggregative games. Consider the following generalized-Cournot game. There are $I$ firms, each choosing their quantity $q_{i} \in X_{i} \subset \mathbb{R}_{+}$. The market price faced by firm $i$ is

$$
p_{i}=P_{i}\left(q_{i}, Q\right)
$$

where

$$
Q: X_{1} \times \ldots \times X_{I} \rightarrow \mathbb{R}_{+}
$$

is an index of aggregate quantity. We assume that $Q$ is separable and strictly increasing in each of its arguments (hence it is an aggregator). Firm $i$ 's profits are

$$
\Pi_{i}\left(q_{i}, Q\right) \equiv \pi_{i}\left(q_{i}, q_{-i}\right)=P_{i}\left(q_{i}, Q\right) q_{i}-c_{i}\left(q_{i}\right)
$$

where $c_{i}\left(q_{i}\right)$ is an increasing function representing firm $i$ 's costs. It is clear that this is an aggregative game.

The most familiar case is that of Cournot competition with homogeneous products, where $Q=\sum_{i=1}^{I} q_{i}$ and $P_{i}\left(q_{i}, Q\right)=P(Q)$. In this case, provided that $P$ is a nonincreasing and concave function of $Q$, this is also a game with strategic substitutes (as defined in Definition 3). Indeed, for $q_{i}^{\prime}>q_{i}$, it is straightforward to verify that

$$
\pi_{i}\left(q_{i:}^{\prime} q_{-i}\right)-\pi_{i}\left(q_{i}, q_{-i}\right)=P\left(Q+q_{i}^{\prime}-q_{i}\right) q_{i}^{\prime}-c_{i}\left(q_{i}^{\prime}\right)-P(Q) q_{i}+c_{i}\left(q_{i}\right)
$$

is decreasing in $q_{j}$ for each $j \neq i$.

It is also straightforward to add a technology choice to this model. This extension is interesting, among other things, for the analysis of technological investments in oligopolistic industries. In particular, suppose that the cost function of each firm is $c_{i}\left(q_{i}, a_{i}\right)$ for some measure of technological investment $a_{i} \in A_{i}$. In addition, there is a cost of technological investment given by $C_{i}\left(a_{i}\right)$. In this case, we have

$$
\prod_{i}\left(q_{i}, a_{i}, Q\right) \equiv \pi_{i}\left(q_{i}, q_{-i}, a_{i}, a_{-i}\right)=P_{\imath}\left(q_{i}, Q\right) q_{i}-c_{i}\left(q_{\imath}, a_{\imath}\right)-C_{\imath}\left(a_{i}\right)
$$

We return to an analysis of this model in Section 6.

\subsection{Bertrand Competition}

Similarly, models of Bertrand competition with differentiated products typically correspond to aggregative games. In such models, firms choose their prices, $p_{i} \in X_{i} \subset \mathbb{R}_{+}$. Suppose that firm $i$ is able to sell quantity $q_{i}$ given by

$$
q_{i}=Q_{i}\left(p_{i}, P\right)
$$


where

$$
P: X_{1} \times \ldots \times X_{I} \rightarrow \mathbb{R}_{+}
$$

is a price index (and again $P$ is separable and strictly increasing in each of its arguments). Firm $i$ profits are given by

$$
\pi_{i}\left(p_{i}, p_{-i}\right) \equiv \Pi_{i}\left(p_{i}, P\right)=p_{i} Q_{i}\left(p_{i}, P\right)-c_{i}\left(Q_{i}\left(p_{i}, P\right)\right)
$$

Clearly, this is again an aggregative game. Perhaps the most commonly studied case is the constant elasticity of demand (Dixit-Stiglitz) model of monopolistic competition, where

$$
P=\left(\sum_{j=1}^{I} p_{j}^{1-\sigma}\right)^{\sigma-1}
$$

and $\sigma$ corresponds to the elasticity of substitution between products in the representative consumers' utility function, while $Q_{i}\left(p_{i}, P\right)=m p_{i}^{-\sigma} P^{\sigma-1}$, with $m>0$, corresponds to the total spending of the representative consumer. The reduced payoff function of firm $i$ can then be written as

$$
\Pi_{i}\left(p_{i}, P\right)=m p_{i}^{1-\sigma} P^{\sigma-1}-c_{i}\left(m p_{i}^{-\sigma} P^{\sigma-1}\right)
$$

\subsection{Patent Races}

The following model of patent races due to Loury (1979) highlights how various different models of innovation can be cast as aggregative games. Suppose that $I$ firms are competing for an innovation and the value of the imovation to firm $i$ is $V_{i} \geq 0$. The model is in continuous time, and each firm chooses a constant $\mathrm{R} \& \mathrm{D}$ intensity at every date, denoted by $s_{i}$. Firm $i$ generates a flow rate of innovation of $h_{i}\left(s_{i}\right)$ as a result of this $R \& D$. The cost of $R \& D$ is $c_{i}\left(s_{i}\right)$. Both $h_{i}$ and $c_{i}$ are increasing functions. The first firm to innovate receives the prize. All firns discount the future at the rate $R>0$. It can be shown that firm $i$ 's expected profits can be written as

$$
\pi_{i}\left(s_{i}, s_{-i}\right)=\int_{0}^{\infty} V_{i} \cdot h\left(s_{i}\right) \cdot \exp \left(-\left(R+\sum_{j=1}^{I} h_{j}\left(s_{j}\right)\right) t\right) d t-c_{i}\left(s_{i}\right) .
$$

It is straightforward to verify that this is an aggregative game with the aggregator given by $g(s)=\sum_{j=1}^{I} h_{j}\left(s_{j}\right)$.

\subsection{Models of Contests and Fighting}

Loury's model, of patent races described in the previous subsection is a special case of models of contests and fighting, for example, as developed by Tullock (1980) and Dixit (1987). These 
models often arise in the context of political economy models or in the analysis of conflict and wars.

Suppose that $I$ players exert effort or invest in their guns or armies in order to win a contest or fight. Again denoting the strategy (corresponding to effort or guns) by $s_{i}$, player $i$ 's payoff can be written as

$$
\pi_{i}\left(s_{i}, s_{-i}\right)=V_{i} \cdot \frac{h_{i}\left(s_{i}\right)}{R+H\left(\sum_{j=1}^{I} h_{j}\left(s_{j}\right)\right)}-c_{i}\left(s_{i}\right),
$$

where again $c_{i}: S_{i} \rightarrow \mathbb{R}_{+}$is the cost function, $h_{1}, \ldots, h_{I}$, and $H$ are strictly increasing functions, and $R \geq 0$. The aggregator in this case is

$$
g(s)=H\left(\sum_{j=1}^{I} h_{j}\left(s_{j}\right)\right),
$$

and reduced payoff functions can be written as

$$
\Pi_{i}\left(s_{i}, Q\right) \equiv \pi_{i}\left(s_{i}, s_{-i}\right)=V_{i} \cdot \frac{h_{i}\left(s_{i}\right)}{R+Q}-c_{i}\left(s_{i}\right) .
$$

It is also useful to note that neither the model of the patent race discussed in the previous subsection nor the more general contest models discussed here are games of strategic substitutes (or complements). For example, suppose that $h$ 's and $H$ are given by the identity function, so that $Q=\sum_{j=1}^{I} s_{j}$. Then, it can be verified that player $i$ will respond to an increase in opponents' strategies by increasing the strategy (strategic complements) if $s_{i} \geq R+\sum_{j \neq i} s_{j}$, while she will decrease her strategy (strategic substitutes) if $s_{i}<R+\sum_{j \neq i} s_{j}$.

\subsection{Private Provision of Public Goods}

Consider the public good provision model of Bergstrom et al. (1986). There are $I$ individuals, each making a voluntary contribution to the provision of a unique public good. Individual $i$ maximizes her utility function

$$
u_{i}\left(c_{i}, \sum_{j=1}^{I} s_{j}+\bar{s}\right)
$$

subject to the budget constraint $c_{i}+p s_{i}=m_{i}$. Here $m_{i}>0$ is income, $c_{i}$ private consumption, and $s_{\imath}$ is agent $i$ 's contribution to the public good, so that $\sum_{j=1}^{I} s_{j}+\bar{s}$ is total amount of the public good provided. The exogenous variable $\bar{s} \geq 0$ can be thought of as the baseline (pre-existing) level of the public good that will be supplied without any contributions.

Substituting for $c_{2}$, it is easily seen that this is an aggregative game with reduced payoff function given by

$$
\Pi_{i}\left(s_{i}, \sum_{j=1}^{I} s_{j}, m, p, \bar{s}\right) \equiv u_{i}\left(m_{i}-p s_{i}, \sum_{j=1}^{I} s_{j}+\bar{s}\right) .
$$


The conditions under which this will also be a game with strategic substitutes are discussed in Section 6.

\subsection{Models with Externalities}

Many models in macroeconomics, environmental economics, search theory, team theory, and other areas feature externalities from some average of the actions of others. ${ }^{10}$ Most generally, in these problems the payoff of player $i$ can be written as $\pi_{i}\left(s_{i}, g(s)\right)$ for some aggregator $g(s)$. Naturally, these games are aggregative. While some such games are also supermodular (for example, when $s_{i}$ and $g(s)$ are "complements"), this is generally not the case. For example, we can think of a situation in which $s_{i}$ corresponds to research effort. Over a certain range, research by others increases the knowledge available to all researchers, so that strategies are complements. However, over a certain other range, various researchers might be competing against each other, so that strategies become substitutes. It is also possible that there might be different types of researchers, such that some of them are "imitators," who benefit from aggregate research, $g(s)$, while others are "innovators," who are, directly or indirectly, harmed by an increase in $g(s)$.

\section{Aggregative Games with Strategic Substitutes}

We first present comparative static results for aggregative games with strategic substitutes (defined in Section 2). ${ }^{11}$ Recall that strategy sets are not one-dimensional, but throughout this section we assume that the aggregate is one-dimensional (i.e., in terms of Definition $1, K=1$ and $N$ is arbitrary). In addition, recall also from Section 2 that throughout payoff functions are upper semi-continuous and strategy sets are compact-but no quasi-concavity or convexity conditions are imposed. The main result of this section is that "regular" comparative statics can be obtained in such games under no additional assumptions. We begin by noting the following:

Theorem 1 (Existence) Let $\Gamma$ be an aggregative game with strategic substitutes. Then $\Gamma$ has a Nash equilibrium (i.e., it has at least one pure-strategy Nash equilibrium).

Proof. See Jensen (2007).

\footnotetext{
${ }^{10}$ See, for example, Diamond (1982) for an early example in the context of search externalities and Cooper and John (1988) for a discussion of a range of macroeconomic models with such externalities.

${ }^{11}$ Note that instead of Definition 3 (supermodularity and decreasing differences), we could equivalently work with quasi-supermodularity and the dual single-crossing property of Milgrom and Shannon (1994). In fact our results will be valid under any set of assumptions that ensure that best-response correspondences are decreasing in the strong set order (e.g., Topkis (1998)). Qnasi-supermodularity and dual single-crossing are ordinal conditions and so hold independently of any strictly increasing transformations of the payoff functions. In particular, a payoff function $\pi_{2}\left(s_{\imath}, s_{-2}, t\right)$ satisfies the dual single-crossing property in $s_{\imath}$ and $s_{-1}$ if for all $\left.s_{2}^{\prime}\right\rangle s_{\imath}$ and $s_{-\imath}^{\prime}\left\langle s_{-\imath}\right.$, (i) $\pi_{i}\left(s_{2}^{\prime}, s_{-2}\right)>\pi_{i}\left(s_{i}, s_{-i}\right) \Rightarrow \pi_{2}\left(s_{i}^{\prime}, s_{-i}^{\prime}\right)>\pi_{2}\left(s_{i}, s_{-i}^{\prime}\right)$, and (ii) $\pi_{2}\left(s_{2}^{\prime}, s_{-2}\right) \geq \pi_{2}\left(s_{i}, s_{-i}\right) \Rightarrow \pi_{2}\left(s_{2}^{\prime}, s_{-2}^{\prime}\right) \geq \pi_{2}\left(s_{2}, s_{-i}^{\prime}\right)$.
} 
Pure-strategy equilibria are not necessarily unique. In general there will be a (compact) set $E(t) \subseteq S$ of equilibria for each parameter $t \in T$. When there are multiple equilibria, we focus on the equilibria with the smallest and largest aggregates. The smallest and largest equilibrium aggregates are defined as

$$
\begin{gathered}
Q_{*}(t) \equiv \min _{s \in E(t)} g(s) \\
Q^{*}(t) \equiv \max _{s \in E(t)} g(s), \text { and }
\end{gathered}
$$

The following theorem establishes certain important properties of the smallest and largest aggregates, which will be used in the subsequent results.

Theorem 2 (Smallest and Largest Aggregates) For all $t \in T, Q_{*}(t)$ and $Q^{*}(t)$ are well defined (i.e., smallest and largest equilibrium aggregates exist). Furthermore the function $Q_{*}$ : $T \rightarrow \mathbb{R}$ is lower semi-continuous, the function $Q^{*}: T \rightarrow \mathbb{R}$ is upper semi-continuous, and thus when there is a unique equilibrium aggregate for all $t, Q_{*}(t)=Q^{*}(t)$ is continuous on $T$.

Proof. See Section 11.1.

Our first substantive result, presented next, addresses the situation where an exogenous parameter $t \in T \subseteq \mathbb{R}$ "hits the aggregator," meaning that it only affects the function $g$. This result is both of substantive interest and also enables us to prove the subsequent characterization results (in Theorems 4 and 5). More formally, we refer to parameter $t$ as a shock to the aggregator (or aggregate) when we can write $\Pi_{i}\left(s_{i}, G(g(s), t)\right) \equiv \pi_{i}(s, t)$ all $i$, where $g: S \rightarrow \mathbb{R}$ designates the aggregator, and $G(g(s), t)$ is continuous, increasing, and separable in $s$ and $t$ (see Definition 1 for the relevant definition of separability). The simplest case would be when the aggregator is linear, so that $\Pi_{i}\left(s_{i}, t+\sum_{j=1}^{I} s_{j}\right) \equiv \pi_{i}(s, t)$ with $G(g(s), t)=t+\sum_{j=1}^{I} s_{j}$ and $g(s)=\sum_{j=1}^{I} s_{j}$. Examples of shocks to the aggregator include a shift in the inverse demand function in the Cournot model (Section 3.1), a change in the discount factor $R$ in a patent race (Section 3.3), or a change in the baseline provision level of the public good $\bar{s}$ in the public good provision model (Section $3.5)$.

Notice that when $t$ is a shock to the aggregator and $t$ is increased, the marginal payoff of each player decreases (provided that marginal payoffs are defined). ${ }^{12}$ Hence we would intuitively expect an increasing shock to the aggregator to lead to a decrease in the aggregate. The next theorem shows that this is indeed the case.

\footnotetext{
${ }^{12}$ By strategic substitutes, agent i's marginal payoff must be decreasing in opponents' strategies and hence, since $G$ is increasing in $s$ and $t$, an increase in $t$ must lead to a decrease in marginal payoff.
} 
Theorem 3 (Shocks to the Aggregator) Consider a shock $t \in T \subseteq \mathbb{R}^{M}$ to the aggregator in an aggregative game with strategic substitutes. Then an increase in $t$ leads to a decrease in the smallest and largest equilibrium aggregates, i.e., the functions $Q_{*}(t)$ and $Q^{*}(t)$ are (globally) decreasing in $t$.

\section{Proof. See Section 11.2.}

Though the result in Theorem 3 is intuitive, we have already seen in Example 1 in the Introduction that such results need not hold in simple games, even in simple aggregative games. In Section 6.3 we present an example of a game with strategic substitutes where a shock leads to a counter-intuitive equilibrium change in the aggregate.

The proof of the theorem exploits the constructive proof of existence of Novshek (1985) (suitably generalized to fit the present framework). This approach provides an explicit description of the largest (and smallest) equilibrium aggregate, allowing us to determine the direction of any change resulting from a shock to the aggregate. We should also add that this approach to comparative statics results is, to the best of our knowledge, new. A major advantage of this approach is that it provides global results that are valid independently of any differentiability and convexity assumptions.

Theorem 3 also allows us to derive a general result on the effect of "entry", i.e., enables a comparison of equilibria when an extra player is added to the game. The entrant, player $I+1$ when the original game has $I$ players, is (by definition) assigned the "inaction" strategy min $S_{I+1}$ before entry (e.g., when $S_{I+1}=[0, \bar{s}]$, inaction corresponds to "zero", $s_{I+1}=0$; for instance, zero production or zero contribution to the provision of a public good). Define the aggregator as $g(s)=g\left(s_{1}, \ldots, s_{I}, s_{I+1}\right)$. Then we have a well-defined aggregative game both before and after entry; before entry there are $I$ players and $s_{I+1}$ is just a constant, after entry this is an $I+1$ player aggregative game in the usual sense. As elsewhere, here increasing means "either strictly increasing or constant". Thus the entrant may choose "inaction" (zero production in the Cournot model, say) and thus the equilibrium after entry may remain the same. ${ }^{13}$

Theorem 4 (Entry) In an aggregative game with strategic substitutes, entry of an additional player will lead to a decrease in the smallest and largest aggregates of the existing players in equilibrium (and a strict decrease if the aggregator $g$ is strictly increasing and the entrant does not choose inaction after entry).

Proof. This result follows from Theorem 3 by observing that the entry of an additional player corresponds to an increasing shock to the aggregate of the existing players. In particular, let

\footnotetext{
${ }^{13}$ To strengthen the results to "strictly increasing," one could impose additional boundary conditions.
} 
$g\left(s_{1}, \ldots, s_{I}, s_{I+1}\right)$ be the aggregator where $I+1$ is the entrant. Since $g$ is separable, we necessarily have $g\left(s_{1}, \ldots, s_{I}, s_{I+1}\right)=H\left(\tilde{g}\left(s_{1}, \ldots, s_{I}\right), s_{I+1}\right)$ where $H$ and $\tilde{g}$ satisfy the above requirements for a shock to the aggregate (see, for example, Vind and Grodal (2003)).

Note that Theorem 4 only shows that the aggregates of the existing players decrease. ${ }^{14}$ It is intuitive to expect that the aggregate inclusive of the entrant should increase. Nevertheless, this is not true without imposing further assumptions (see Remark 9 in the proof of Theorem 3 for a detailed description of when entry will decrease the aggregate). In the next section, we will show that, under additional assumptions, entry can also be shown to increase the overall aggregate (see Theorem 8).

The next theorem presents our most powerful results for games with strategic substitutes. These can be viewed as the strategic substitutes counterparts of the monotonicity results that are well-known for supermodular games. One difference, however, is that with strategic substitutes, the results apply only when shocks are idiosyncratic, i.e., to shocks $t_{i}$ that affect only a single player, $i \in I$. More formally, a change in $t_{i}$ is an idiosyncratic shock to player $i$ if payoff functions can be written as

$$
\begin{aligned}
\pi_{i}\left(s, t_{i}\right) & \equiv \Pi_{i}\left(s_{i}, g(s), t_{i}\right), \text { and } \\
\pi_{j}\left(s, t_{i}\right) & \equiv \Pi_{j}\left(s_{j}, g(s)\right) \text { for all } j \neq i .
\end{aligned}
$$

Let us also introduce the notion of a positive shock.

Definition 5 (Positive Shock) Consider the payoff function $\pi_{i}=\pi_{i}\left(s_{i}, s_{-i}, t_{i}\right)$. Then an increase in $t_{i}$ is a positive shock if $\pi_{i}$ exhibits increasing differences in $s_{i}$ and $t$.

It is straightforward to verify that Definition 5 gives the correct notion of "positive shock"; $\pi_{i}$ exhibits increasing differences if only if player $i$ 's "marginal payoff", $\pi_{i}\left(s_{i}^{\prime}, s_{-i}, t\right)-\pi_{i}\left(s_{i}, s_{-i}, t\right)$ for $s_{i}^{\prime}>s_{i}$, is increasing in $t$. Moreover, as is well known, when $\pi_{i}$ is sufficiently smooth, it will exhibit increasing differences in $s_{i}$ and $t$ if and only if the cross-partial is nonnegative, i.e., $D_{s_{2} t}^{2} \pi \geq 0$ for all $s$ and $t$. The single-crossing property may replace increasing differences in the previous definition without changing any of our results. We also define smallest and largest equilibrium strategies for player $i$ analogously to the smallest and largest equilibrium aggregates.

Theorem 5 (Idiosyncratic Shocks) Let $t_{i}$ be a positive idiosyncratic shock to player $i$. Then an increase in $t_{i}$ leads to an increase in the smallest and largest equilibrium strategies for player

\footnotetext{
${ }^{14}$ This is the reason why we do not explicitly write $Q^{*}(t)$ and $Q \cdot(t)$. Instead, we could have defined $\tilde{Q}^{*}(t) \equiv$ $\max _{\left(s_{1}, \ldots, s_{l}\right) \in E(t)} \tilde{g}\left(s_{1}, \ldots, s_{l}\right)$ and $\tilde{Q}_{*}(t) \equiv \min _{\left(s_{1}, \ldots, s_{I}\right) \in E(t)} \bar{g}\left(s_{1}, \ldots, s_{I}\right)$, and the statement would be for $\bar{Q}^{*}(t)$ and $\tilde{Q} .(t)$. But this additional notation is not necessary for the statement or the proof of the theorem.
} 
$i$, and to a decrease in the associated aggregates of the remaining players (which are, respectively, the largest and smallest such aggregates).

Proof. See Section 11.3.

A simple corollary to Theorem 5 also characterizes the effects of a positive shock on payoffs.

Corollary 1 (Payoff Effects) Assume in addition to the conditions of Theorem 5 that all payoff functions are decreasing [respectively, increasing] in opponents'strategies and that player i's payoff function is increasing [respectively, decreasing] in the idiosyncratic shock $t_{i}$. Then an increase in $t_{i}$ increases [respectively, decreases] player $i$ 's payoff in equilibrium and decrease /respectively, increases] the payoff of at least one other player.

Proof. For player $i, \pi_{i}\left(s_{i}^{\prime}, g\left(s^{\prime}\right), t^{\prime}\right) \leq \pi_{i}\left(s_{i}^{\prime}, g\left(s_{i}^{\prime}, s_{-i}^{\prime \prime}, t^{\prime \prime}\right) \leq \pi_{i}\left(s_{i}^{\prime \prime}, g\left(s^{\prime \prime}\right), t^{\prime \prime}\right)\right.$. Since the strategy of some player $j$ (for $j \neq i$ ) decreases, we must have $\sum_{k \neq j} h_{k}\left(s_{k}^{\prime}\right) \leq \sum_{k \neq j} h_{k}\left(s_{k}^{\prime \prime}\right)$. Consequently, $\pi_{j}\left(s_{j}^{\prime \prime}, g\left(s^{\prime \prime}\right)\right) \leq \pi_{j}\left(s_{j}^{\prime \prime}, g\left(s_{j}^{\prime \prime}, s_{-j}^{\prime}\right)\right) \leq \pi_{j}\left(s_{j}^{\prime}, g\left(s^{\prime}\right)\right)$.

\section{$5 \quad$ Nice Aggregative Games}

We now extend the framework of the previous section to aggregative games without strategic substitutes. For this purpose, we focus on "nice" games where payoff functions are differentiable and concave (or pseudo-concave) in own strategies. ${ }^{15}$ We also impose an additional local solvability condition. As in the previous section, we focus on games where the aggregator is real valued (i.e., $K=1$ in terms of Definition 1).

The following definition introduces the notion of "nice" aggregative games formally. Although we include a boundary condition as part of this definition, when strategy sets are one-dimensional this can be replaced by a weaker "regularity" condition (see Definition 8).

Definition 6 (Nice Aggregative Games) An aggregative game $\Gamma$ is said to be a nice aggregative game if the aggregator $g$ is twice continuously differentiable, each strategy set is compact and convex, and every payoff function $\pi_{i}$ is twice continuously differentiable, and pseudo-concave in the player's own strategies. Furthermore, we have that whenever $s_{i} \in \partial S_{i}$ (with $\partial S_{i}$ denoting the boundary of the strategy set $\left.S_{i}\right)$ and $\left(v-s_{i}\right)^{T} D_{s_{i}} \pi_{i}(s, t) \leq 0$ for all $v \in S_{i}$, then $D_{s_{i}} \pi_{i}(s, t)=0$. That is, the first-order conditions $D_{s_{i}} \pi_{i}(s, t)=0$ are required to hold whenever a boundary strategy for player $i$ is a (local) best response.

\footnotetext{
${ }^{15}$ Weinstein and Yildiz (2008) use a similar definition of a "nice game," except that they also impose onedimensional strategies and concavity (rather than pseudo-concavity).
} 
Remark 3 (Pseudo-concavity) Recall that a differentiable function $\pi_{i}$ is pseudo-concave (Mangasarian (1965)) in $s_{i}$ if for all $s_{i}, s_{i}^{\prime} \in S_{i}$ :

$$
\left(s_{i}^{\prime}-s_{i}\right)^{T} D_{s_{\imath}} \pi_{i}\left(s_{i}, s_{-i}, t\right) \leq 0 \Rightarrow \pi_{i}\left(s_{i}^{\prime}, s_{-i}, t\right) \leq \pi_{i}\left(s_{i}, s_{-i}, t\right) .
$$

Naturally, any concave function is pseudo-concave. Pseudo-concavity implies that the first-order conditions $D_{s_{i}} \pi_{i}(s, t)=0$ are sufficient for $s_{i}$ to maximize $\pi_{i}$ given $s_{-i}$ and $t$. That first-order conditions are sufficient for a maximum is what we use in the proofs. Pscudo-concavity is not a necessary condition for this to hold. For example, if $N=1$ and $D_{s_{i}} \pi_{i}(s, t)=0 \Rightarrow D_{s_{2} s_{i}}^{2} \pi_{i}(s, t)<0$, it is easy to sce that the first-order condition will be sufficient for a maximum (and in fact, that the maximum will be unique). Quasi-concavity (or even strict quasi-concavity) does not imply the sufficiency of first-order conditions for a maximum in general.

Remark 4 (Inada-Type Boundary Conditions) Note also that the boundary condition in Definition 6 does not rule out best responses on the boundary of a player's strategy set, $\partial S_{i}$. Instead, it simply requires first-order conditions to be satisfied whenever a local best response is on the boundary. Consequently, this boundary condition is weaker than the standard "Inadatype" conditions ensuring that best responses alway's lie in the interior of strategy sets (since when best responses never lie on the boundary, first-order conditions vacuously hold for best responses on the boundary). ${ }^{16}$

As is well-known, the concavity or pseudo-concavity conditions ensure that best response correspondences are convex valued (they are also upper hemi-continuous as mentioned at the beginning of Section 2). The existence of a pure-strategy Nash equilibrium therefore follows by Kakutani's fixed point theorem. ${ }^{17}$ For the record, we note this result in the following theorem (proof omitted).

Theorem 6 (Existence) Suppose that $\Gamma$ is a nice aggregative game. Then $\Gamma$ has a (purestrategy) Nash equilibrium.

\footnotetext{
${ }^{16}$ However, all boundary conditions cannot be dispensed with. To see this, consider an $N$-dimensional game, $N>1$ (with each player having $N$-dimensional strategy sets) without any interior best responses. The boundary of this $N$-dimensional game can then be mapped bijectively into an $N$ - 1-dimensional game. But since first-order conditions never have to hold in the $N$-dimensional game, the local solvability condition below (Definition 7 ) would never have to hold. In effect, the $N$-1-dimensional "reduction" is therefore unrestricted and consequently, no general results can be derived for such a game.

${ }^{17}$ Without convex best response correspondences, a Nash equilibrium may fail to exist in an aggregative game (unless the game also features strategic substitutes or complements). See Jensen (2007), Example 5 for an example of an aggregative game where a pure-strategy Nash equilibrium fails to exist even though strategy sets are onedimensional, convex, and there are only two players.
} 
A nice aggregative game does not necessarily have a unique equilibrium. Similarly, the local solvability condition introduced below does not guarantee uniqueness. We therefore deal with the possible multiplicity of equilibria as in the previous section and study the behavior of the smallest and largest equilibrium aggregates, $Q_{*}(t)$ and $Q^{*}(t)$. Theorem 2 from the previous section still applies and the smallest and largest equilibrium aggregates continue to exist and are, respectively, lower and upper semi-continuous, in $t$.

We next introduce the local solvability condition, which will play a central role in our analysis in this section. Let us simplify notation by defining $D_{1} \Pi_{i}\left(s_{i}, Q, t\right) \equiv D_{s_{i}} \Pi_{i}\left(s_{i}, Q, t\right)$ and $D_{2} \Pi_{i}\left(s_{i}, Q, t\right) \equiv D_{Q} \Pi_{i}\left(s_{i}, Q, t\right)$. Using the fact that $g$ is a twice continuously differentiable aggregator, the marginal payoff for player $i$ can be expressed as:

$$
D_{s_{i}} \pi_{i}(s, t)=D_{1} \Pi_{i}\left(s_{i}, g(s), t\right)+D_{2} \Pi_{\imath}\left(s_{i}, g(s), t\right) f_{i}\left(s_{i}, g(s)\right)
$$

Where $f_{i}\left(s_{i}, g(s)\right)=D_{s_{i}} g(s)($ cf. Remark 1).

Equation (7) shows that the marginal payoff is a function of the player's own strategy $s_{i}$ and the aggregate $g(s)$. Let us also define a function $\Psi_{i}: S_{i} \times \mathbb{R} \times T \rightarrow \mathbb{R}^{N}$ that makes this dependence explicit:

$$
\Psi_{i}\left(s_{i}, Q, t\right) \equiv D_{1} \Pi_{i}\left(s_{i}, Q, t\right)+D_{2} \Pi_{i}\left(s_{i}, Q, t\right) f_{i}\left(s_{i}, Q\right) .
$$

Note that this function contains the same information as (7), though it also enables us to separate the direct and indirect effects of $s_{i}$ (the first one corresponding to a change in $\Psi_{i}$ holding $Q$ constant). Naturally,

$$
\Psi_{i}\left(s_{i}, Q\right)=0 \Leftrightarrow\left[D_{s_{i}} \pi_{i}(s, t)=0 \text { and } g(s)=Q\right] .
$$

Differentiating $\Psi_{i}$ with respect to $s_{i}$ yields an $N \times N$ matrix $D_{s_{2}} \Psi_{i}\left(s_{i}, Q, t\right) \in \mathbb{R}^{N \times N}$. The determinant of this matrix is denoted by $\left|D_{s_{i}} \Psi_{i}\left(s_{i}, Q, t\right)\right| \in \mathbb{R}$. If strategy sets are one-dimensional, $\left|D_{s_{i}} \Psi_{i}\left(s_{i}, Q, t\right)\right|=D_{s_{2}} \Psi_{i}\left(s_{i}, Q, t\right) \in \mathbb{R}$.

We now introduce a key concept for the study of comparative statics of nice games.

Definition 7 (Local Solvability) Player $i \in \mathcal{I}$ is said to satisfy the local solvability condition if $\left|D_{s_{i}} \Psi_{i}\left(s_{i}, Q, t\right)\right| \neq 0$ whenever $\Psi_{i}\left(s_{i}, Q, t\right)=0$ (for $s_{i} \in S_{i}, Q \in\{g(s): s \in S\}$ ).

The local solvability condition requires that the determinant of $D_{s_{i}} \Psi_{i}$ is nonzero on the subspace where $\Psi_{i}=0$. The term refers to the fact that if this determinant were equal to zero, there would not be a unique solution to a set of equations of the form $D_{s_{2}} \Psi_{i} \cdot a=b$ (again defined on the subspace where $\Psi_{i}=0$ ), where $a$ and $b$ are $N$-dimensional vectors. This type of equation 
arises when constructing the backward reply correspondences for the purposes of comparative static analysis. This discussion also motivates the term "local solvability condition".

We may now also state a weaker boundary condition that can replace, when $N=1$, the one in Definition 6.

Definition 8 (Regularity) For $N=1$ we say that $\Psi_{i}$ is regular if, for any $t \in T$, there does not exist a $Q \in X$ such that $D_{s_{i}} \Psi_{i}\left(s_{i}, Q, t\right)>0$ for all $s_{i} \in\left\{s_{i}: \Psi_{i}\left(s_{i}, Q, t\right)=0\right\}$.

Given the relationship between $\Psi_{i}$ and $D_{s_{i}} \pi_{i}$ in (8), $D_{s_{2}} \Psi_{i}>0$ on the subspace where $\Psi_{i}=0$ can be viewed as "perverse". The regularity conditions in Definition 8 rules out such perverse cases and is straightforward to check.

Remark 5 (Ordinality) It also useful to note that local solvability condition is ordinal: it is independent of the choice of the coordinate system and holds for any strictly increasing transformation of the aggregator. Firstly, local solvability holds for the payoff function $\pi_{i}(s, t)$ if and only if it holds for $\Phi\left(\pi_{i}(s, t)\right)$ where $\Phi: \mathbb{R} \rightarrow \mathbb{R}$ is any strictly increasing and twice continuously differentiable function, with derivative denoted by $\Phi^{\prime}$ (where differentiability is needed here to ensure that the transformed payoff function is also twice continuously differentiable). In particular, for all $s_{i}^{\prime}$ and $Q^{\prime}$, we have that

$$
\Psi_{i}\left(s_{i}^{\prime}, Q^{\prime}, t\right)=0 \Leftrightarrow \Phi^{\prime}\left(\Pi_{i}\left(s_{i}, Q, t\right)\right) \Psi_{i}\left(s_{i}, Q, t\right)=0
$$

Ordinality of the local solvability condition follows if $\left|D_{s_{i}} \Psi_{i}\left(s_{i}^{\prime}, Q^{\prime}\right)\right| \neq 0$ implies $\left|D_{s_{i}}\left[\Phi^{\prime}\left(\Pi_{i}\left(s_{i}^{\prime}, Q^{\prime}, t\right)\right) \Psi_{i}\left(s_{i}^{\prime}, Q^{\prime}, t\right)\right]\right| \neq 0$. This is true since, when $\Psi_{i}\left(s_{i}^{\prime}, Q^{\prime}, t\right)=0$, $\left|D_{s_{2}}\left[\Phi^{\prime}\left(\Pi_{i}\left(s_{i}^{\prime}, Q^{\prime}, t\right)\right) \Psi_{i}\left(s_{i}^{\prime}, Q^{\prime}, t\right)\right]\right|=\Phi^{\prime}\left(\Pi_{i}\left(s_{i}^{\prime}, Q^{\prime}, t\right)\right)\left|D_{s_{i}} \Psi_{i}\left(s_{i}^{\prime}, Q^{\prime}\right)\right|$.

Secondly, the local solvability condition is also independent of the coordinate system. In particular, we can replace each strategy vector $s_{i}$ by transformed variable vector $\tilde{s}_{i}=\psi_{i}\left(s_{i}\right)$ where $\psi_{i}: \mathbb{R}^{N} \rightarrow \mathbb{R}^{N}$ is a diffeomorphism for each $i$. The payoff function of player $i$ then becomes $\pi_{i}\left(\psi_{i}^{-1}\left(\tilde{s}_{i}\right), \psi_{-i}^{-1}\left(s_{-i}\right), t\right)$, where $\psi_{-i}^{-1}=\left(\psi_{j}^{-1}\right)_{j \neq i}$. It can be verified that $\pi_{i}$ satisfies the local solvability condition in $s$ (in the original variables) if and only if the same condition is satisfied for any such (smooth) change of variables. In particular, local solvability in the new coordinate system requires

$$
D \psi_{i}^{-1}\left(\tilde{s}_{i}\right) \Psi_{i}\left(\psi_{i}^{-1}\left(\tilde{s}_{i}\right), Q\right)=0 \Rightarrow\left|D_{\tilde{s}_{i}}\left[D \psi_{i}^{-1}\left(\tilde{s}_{i}\right) \Psi_{i}\left(\psi_{i}^{-1}\left(\tilde{s}_{\imath}\right), Q\right\rangle\right]\right| \neq 0
$$

Since $D \psi_{i}^{-1}\left(\tilde{s}_{i}\right) \Psi_{i}\left(\psi_{i}^{-1}\left(\tilde{s}_{i}\right), Q\right)=0 \Leftrightarrow \Psi_{i}\left(\psi_{i}^{-1}\left(\tilde{s}_{i}\right), Q\right)=0\left(D \psi_{\imath}^{-1}\left(\tilde{s}_{i}\right)\right.$ is a full rank matrix), we have that

$$
\left|D_{\tilde{s}_{\imath}}\left[D \psi_{i}^{-1}\left(\tilde{s}_{i}\right) \Psi_{\imath}\left(\psi_{i}^{-1}\left(\tilde{s}_{i}\right), Q\right)\right]\right|=\left|D \psi_{i}^{-1}\left(\tilde{s}_{\imath}\right) D_{s_{\imath}} \Psi_{\imath}\left(\psi_{i}^{-1}\left(\tilde{s}_{i}\right), Q\right)\left[D \psi_{i}^{-1}\left(\tilde{s}_{i}\right)\right]^{T}\right|
$$


This establishes the desired result.

That local solvability is independent of any strictly increasing transformation of the aggregator implies that instead of the aggregator $g(s)$ we may use any aggregator $\tilde{g}(s)=f(g(s)$ ) where $f: \mathbb{R} \rightarrow \mathbb{R}$ is a strictly increasing and differentiable function. To see this simply note that $\Pi_{i}\left(s_{i}, g(s)\right)=\Pi_{i}\left(s_{i}, f^{-1}(\tilde{g}(s))\right)$ where $\tilde{g}(s)=f(g(s))$. Denoting the new aggregate by $\tilde{Q}=\tilde{g}(s)$, it is clear that $\Psi_{i}\left(s_{i}, \tilde{Q}\right)=\Psi_{i}\left(s_{i}, Q\right)$. Evidently then $\left|D_{s_{i}} \Psi_{i}\left(s_{i}, Q\right)\right|=\left|D_{s_{i}} \Psi_{i}\left(s_{i}, \tilde{Q}\right)\right|$ and the conclusion follows.

Remark 6 (Weaker Conditions) Some version of the local solvability condition cannot be dispensed with. Example 1 in the Introduction shows the possibility of perverse comparative statics when the local solvability condition does not hold (see also Section 6.3). Nevertheless, the results presented in this section continue to hold under weaker conditions. In particular, the following generalization would be sufficient, though the condition in Definition 7 is easier to state and verify. The alternative condition is as follows: when $\Psi_{i}\left(s_{i}, Q, t\right)=0$, there should be open neighborhoods $\mathcal{N}_{s_{i}}$ and $\mathcal{M}_{Q}$ of $s_{i}$ and $Q$, respectively, and a continuous map $b_{i}: \mathcal{M}_{Q} \rightarrow \mathcal{N}_{s_{i}}$ such that for each $\hat{Q} \in \mathcal{M}_{Q}, b_{i}(\hat{Q})$ is the unique solution to $\Psi_{i}\left(s_{i}, \hat{Q}, t\right)=0$ in $\mathcal{N}_{s_{2}}$. This implies that that the first-order condition $\Psi_{i}\left(s_{i}, Q, t\right)=0$ admits a local solution in $s_{i}$ as a function of Q. Naturally, in view of the implicit function theorem, this weaker condition follows from our local solvability condition in Definition 7. Other alternatives to the local solvability condition are discussed in Section 8.

We next introduce the appropriate notion of positive slıccks for nice games. This generalizes the notion of a positive shock introduced in Definition 5. Because the aggregator is separable it can be written as $g(s)=H\left(\sum_{j=1}^{I} h_{j}\left(s_{j}\right)\right)$ where $h_{i}: S_{i} \rightarrow \mathbb{R}$ and $H: \mathbb{R} \rightarrow \mathbb{R}$ (cf. Definition 1). It is clear that the term $h_{i}\left(s_{i}\right)$ fully "captures" agent $i$ 's effect on the aggregate. Intuitively, our generalized definition of a positive shock requires that an increase in the exogenous variable leads to an increase in the term $h_{i}\left(s_{i}\right)$ and thus increases the aggregate given everyone else's strategies. In comparison, our previous definition, Definition 5, made the stronger requirement that a player's (smallest and largest) strategy should increase with the exogenous variable.

Definition 9 (Positive Shocks) A change in the parameter vector $t$ is a positive shock to player $i$ if the largest and smallest selections from this player's "composite" best responds correspondence $h_{i}\left(R_{i}\left(s_{-i}, t\right)\right)$ are increasing in $t$. That is, consider $t<t^{\prime} \in T$ and let $\bar{h}_{i} \circ r_{i}\left(s_{-i}, t\right)$ and $\underline{h}_{i} \circ r_{i}\left(s_{-i}, t\right)$ be the maximal and minimal elements of $h_{i}\left(R_{\imath}\left(s_{-i}, t\right)\right) \subseteq \mathbb{R}$. Then $t$ is a positive shock to player $i$ if and only if $\overline{h i}_{i} \circ r_{i}\left(s_{-i}, t\right) \leq \bar{h}_{i} \circ r_{i}\left(s_{-i}, t^{\prime}\right)$ and $\underline{h}_{i} \circ r_{i}\left(s_{-i}, t\right) \leq \underline{h_{i} \circ r_{i}}\left(s_{-i}, t^{\prime}\right)$ for all $s_{-i} \in S_{-2}$. 
Note that if a player $i$ 's strategy set is a lattice, his payoff function is supermodular in $s_{i}$, and exhibits increasing differences in $s_{i}$ and $t$, then the shock will be positive. But in general, a positive shock need not satisfy these "supermodularity" conditions. For example, a shock $t$ is a positive shock for a player when it does not affect the player's payoff function (regardless of any lattice or supermodularity conditions).

Our first result in this section characterizes the comparative statics of the aggregate and strengthens Theorem 3 from the previous section. In particular, in contrast to Theorem 3 , this results applies whether or not the shock hits the aggregate, and it also applies whether or not the shock hits one, two, or all of the players.

Theorem 7 (Aggregate Comparative Statics) Consider a nice aggregative game where each player's payoff function satisfies the local solvability condition. Then a positive shock $t \in T$ leads to an increase in the smallest and largest equilibrium aggregates, i.e., the functions $Q_{*}(t)$ and $Q^{*}(t)$ are (globally) increasing in $t$.

The result remains valid without any boundary conditions on payoff functions when $N=1$ and $\Psi_{i}$ is regular.

\section{Proof. See Section 11.4}

Our next result extends Theorem 4 in the previous section. In particular, it strengthens Theorem 4 to a statement for the overall aggregates (after the entry of the new additional player instead of the aggregates of the strategies of existing players). Let us define $0 \in S_{i}$ to stand for "inaction". As in the previous section, the convention is that $I+1$ th player takes this action before entry.

Theorem 8 (Entry) Let $Q_{*}(I)$ and $Q^{*}(I)$ denote the smallest and largest equilibrium aggregates in a game with $I \in \mathbb{N}$ players that satisfies the conditions of Theorem 7 and where $S_{i} \subseteq \mathbb{R}_{+}^{N}$, $0 \in S_{i}$ for all $i \in \mathcal{I}$. Then for any $I \in \mathbb{N}, Q_{*}(I) \leq Q_{*}(I+1)$ and $Q^{*}(I) \leq Q^{*}(I+1)$, i.e., entry increases the smallest and largest aggregates in equilibrium. Moreover, if the aggregator $g$ is strictly increasing and the entrant chooses a non-zero strategy following entry, the above inequalities are strict.

Proof. See Section 11.5.

Finally, our third result strengthens Theorem 5 from the previous section and characterizes the comparative statics of individual strategies. It is useful to note Theorem 9 is the first (and only) result among those presented in this and the previous section that uses the implicit function 
theorem. As such it is a purely local result and also requires that the equilibrium strategy studied is interior.

Theorem 9 (Individual Comparative Statics) Let the "conditions of Theorem 7 be satisfied and consider player $i$ 's equilibrium strategy $s_{i}^{*}(t)$ associated with the smallest (or largest) equilibrium aggregate at some equilibrium $s^{*}=s^{*}(t)$ given $t \in T$. Assume that the equilibrium $s^{*}$ lies in the interior of $S$ and that $t$ is a positive shock. Then the following results hold.

- $s_{i}^{*}(t)$ is (coordinatewise) locally increasing in $t$ provided that

$$
-\left[D_{s_{i}} \Psi_{i}\left(s_{i}^{*}, g\left(s^{*}\right), t\right)\right]^{-1} D_{Q} \Psi_{i}\left(s_{i}^{*}, g\left(s^{*}\right), t\right) \geq 0
$$

- Suppose that the shock $t$ does not directly affect player $i$ (i.e., $\pi_{i}=\pi_{i}(s)$ ). Then the sign of each element of the vector $D_{t} s_{i}^{*}(t)$ is equal to the sign of each element of the vector - $\left[D_{s_{i}} \Psi_{i}\left(s_{i}^{*}, g\left(s^{*}\right)\right)\right]^{-1} D_{Q} \Psi_{i}\left(s_{i}^{*}, g\left(s^{*}\right)\right)$. In particular, $s_{i}^{*}(t)$ will be (coordinatewise) locally decreasing in $t$ whenever:

$$
-\left[D_{s_{i}} \Psi_{i}\left(s_{i}^{*}, g\left(s^{*}\right)\right)\right]^{-1} D_{Q} \Psi_{i}\left(s_{i}^{*}, g\left(s^{*}\right)\right) \leq 0
$$

Proof. By the implicit function theorem, we have

$$
D_{s_{i}} \Psi_{i}\left(s_{i}, Q, t\right) d s_{i}=-D_{Q} \Psi_{i}\left(s_{i}, Q, t\right) d Q-D_{s_{i} t}^{2} \Pi_{i}\left(s_{i}, Q, t\right) d t
$$

The results follow from this observation and the fact that $Q$ increases with $t$ (where $Q$ is either the smallest or largest equilibrium aggregate).

\section{Applying the Theorems}

In this section, we return to some of the examples presented in Section 3 and show how the results of the last two sections can be applied to obtain sharp comparative static results. We first discuss private provision of public goods and contests, and then turn to a game of technology choices in an oligopolistic setting, which also illustrates how these results can be applied when strategies are multi-dimensional.

\subsection{Private Provision of Public Goods}

In Bergstrom et al. (1986)'s public good provision model described in Section 3.5, agent i's reduced utility function was given by: 


$$
\Pi_{i}\left(s_{i}, \sum_{j=1}^{I} s_{j}, m, p, \bar{s}\right) \equiv u_{i}\left(m_{i}-p s_{i}, \sum_{j=1}^{I} s_{j}+\bar{s}\right)
$$

As mentioned in Section 3.5, the aggregator is here simply $g(s)=\sum_{j=1}^{I} s_{j}$. When $s^{*}=\left(s_{i}^{*}\right)_{i \in \mathcal{I}}$ is an equilibrium, we refer to $g\left(s^{*}\right)=\sum_{i=1}^{I} s_{i}^{*}$ as the aggregate equilibrium provision. Let us simplify the exposition and notation here by assuming that $u_{i}$ is smooth and that strategy sets are intervals of the type $S_{i}=\left[0, \bar{s}_{i}\right] \subseteq \mathbb{R}$. The private good will be normal if and only if the following condition holds for all $s \in S$ :

$$
-p D_{12}^{2} u_{i}\left(m_{i}-p s_{i}, \sum_{j=1}^{I} s_{j}+\bar{s}\right)+D_{22}^{2} u_{i}\left(m_{i}-p s_{i}, \sum_{j=1}^{I} s_{j}+\bar{s}\right) \leq 0 .
$$

Notice also that the left-hand side of $(9)$ is equal to $D_{s_{2} s_{j}}^{2} \Pi_{i}$. Therefore, whenever the private good is normal, payoff functions exhibit decreasing differences and this is a game with strategic substitutes (cf. Definition 3). The following result therefore follows directly from the results in Section 4 (proof omitted):

Proposition 1 Consider the public good provision game presented in Section 3.5 and assume that the private good is normal. Then there exists a (pure-strategy) Nash equilibrium. Furthermore:

1. An increase in $\bar{s}$ leads to a decrease in the smallest and largest aggregate equilibrium provisions.

2. The entry of an additional agent leads to a decrease in the smallest and largest aggregate equilibrium provisions by existing agents.

3. A positive shock to agent $i$ will lead to an increase in that agent's smallest and largest equilibrium provisions and to a decrease in the associated aggregate provisions of the remaining $I-1$ players.

Proposition 1 holds under the assumption that the private good is normal and exploits the results from Section 4. Alternatively, we can use the results from Section 5 when the public good is (strictly) normal. This is illustrated next. Suppose that the payoff function is pseudo-concave (which was not assumed for Proposition 1). Then the public good will be (strictly) normal if and only if

$$
D_{s_{\imath}} \Psi_{i}\left(s_{i}, Q\right)=p^{2} D_{11} u_{\imath}\left(m_{i}-p s_{i}, Q\right)-p D_{21} u_{i}\left(m_{\imath}-p s_{i}, Q\right)<0
$$


for all $s \in S .{ }^{18}$ Moreover, whenever (10) holds, an increase in $m_{i}$ (or a decrease in $p$ ) constitutes a positive shock, i.e., $D_{s_{i} m}^{2} \Pi_{i} \geq 0$ and $D_{s_{i} p}^{2} \Pi_{i} \leq 0$, respectively (cf. Definition 9 ). The next proposition then follows from Theorems 7-9 (proof omitted). ${ }^{19}$

Proposition 2 Consider the public good provision game presented in Section 3.5 and assume that the public good is (strictly) normal, that payoff functions are pseudo-concave in own strategies and that strategy sets are convex. Then there exists a (pure-strategy) Nash equilibrium. Furthermore:

1. Any positive shock to one or more of the agents (e.g., a decrease in p, or increases in one or more income levels, $m_{1}, \ldots, m_{J}$ ) leads to an increase in the smallest and largest aggregate equilibrium provisions.

2. The smallest and largest aggregate equilibrium provisions are increasing in the number of agents.

3. The changes in 1 and 2 above are associated with an increase in the provision of agent $i$ if the private good is inferior for this agent, and with a decrease in agent $i$ 's provision if the private good is normal and the shock does not directly affect the agent.

It is also useful to note that Proposition 2 could be obtained even under weaker conditions by using Corollary 3 presented in Section 8 below. In particular, it can be verified that if the public good is normal (condition (10) holding as weak inequality) and payoff functions are quasi-concave (rather than pseudo-concave), the conditions of this corollary are satisfied and Proposition 2 remains valid. We used Theorems $7-9$ here since Corollary 3 is not introduced until Section 8.

\subsection{Models of Contests and Fighting}

Recall that the payoff function of a participant in a contest can be written as

$$
\pi_{i}\left(s_{i}, s_{-i}\right)=V_{i} \cdot \frac{h_{i}\left(s_{i}\right)}{R+H\left(\sum_{j=1}^{I} h_{j}\left(s_{j}\right)\right)}-c_{i}\left(s_{i}\right),
$$

where $s_{i}$ denotes agent $i$ 's effort, $h_{i}: \mathbb{R}_{+} \rightarrow \mathbb{R}_{+}$for each $i \in \mathcal{I}$ and $H: \mathbb{R}_{+} \rightarrow \mathbb{R}_{+}$. As mentioned in Section 3.4, contests generally feature neither strategic substitutes nor complements. Therefore, the results in Section 4 do no apply, nor do any of the well-known results on supermodular games mentioned in the Introduction. In this case, the most obvious strategy for deriving comparative

\footnotetext{
${ }^{18}$ The equivalence between (strict) normality of the public good and (10) follows since $\partial_{s_{\imath}}\left(m, p, \sum_{j \neq i} s_{j}\right) / \partial m=$ $\alpha\left(p D_{12}^{2} u_{2}-p^{2} D_{11}^{2} u_{i}\right)$.

${ }^{19}$ Note in particular that (strict) normality implies local solvability as well as regularity so the statements in Proposition 2 are valid without any boundary conditions on payoff functions.
} 
static results is to use the implicit function theorem. This is indeed what most of the literature does. Nevertheless, the implicit function theorem also runs into difficulties unless we make additional, strong assumptions. For this reason, previous treatments have restricted attention to special cases of the above formulation. For example, Tullock (1980) studied two-player contests, while Loury (1979) focused on symmetric contests with (ad hoc) stability conditions. More recently, Nti (1997) provided comparative statics results in the (symmetric) Loury model of patent races, assuming that $H=i d$ (the identity function), $h_{i}=h$ for all $i$ and concave, and linear costs. Using the results of Section 5 , we can establish considerably more general and robust results on contests and fighting games.

These results are provided in the following proposition (and also generalize the existence result of Szidarovszky and Okuguchi (1997)).

Proposition 3 Consider the contest games introduced in Section 3.4 and suppose that $H$ is convex, $h_{i}$ and $c_{i}$ are strictly increasing, and that the following condition holds:

$$
\frac{h_{i}^{\prime \prime}\left(s_{i}\right)}{h_{i}^{\prime}\left(s_{i}\right)} \leq \frac{c_{i}^{\prime \prime}\left(s_{i}\right)}{c_{i}^{\prime}\left(s_{i}\right)} \text { for all } s_{i} \in S_{i} .
$$

Then there exists a (pure-strategy) Nash equilibrium. Furthermore:

1. The smallest and largest aggregate equilibrium efforts are increasing in any positive shock (e.g., a decrease in $R$ or an increase in $V_{i}$ for one or more players).

2. Entry of an additional player increases the aggregate equilibrium effort.

3. There exists a function $\eta: \mathbb{R} \rightarrow \mathbb{R}$ such that the changes in parts 1 or 2 above are associated with an increase in the effort of player $i \in \mathcal{I}$ and the corresponding equilibrium aggregate $Q^{*}$ provided that $i$ is "dominant" in the sense that $h_{i}\left(s_{i}^{*}\right) \geq \eta\left(Q^{*}\right)$. Conversely, if $i$ is "not dominant", i.e., $h_{i}\left(s_{i}^{*}\right)<\eta\left(Q^{*}\right)$, then the changes in parts 1 and 2 decrease player $i$ 's effort provided that the shock does not affect this player directly (e.g., corresponding to a decrease in another player's costs).

Proof. See Section 11.6

This proposition can also be extended to the case in which $H$ is not convex. Convexity of $H$ ensures that the first-order condition $D_{s_{i}} \pi_{i}\left(s_{i}, s_{-i}\right)=0$ is sufficient for a maxinuum, but it is not necessary for this conclusion. Observe also that the conditions of Proposition 3 are satisfied if $H$ is the identity function, $c_{i}$ is convex, and $h_{\imath}$ is concave. ${ }^{20}$ Szidarovszky and Okuguchi

\footnotetext{
${ }^{20}$ The proof of Proposition 3 shows that the function $\eta$ in part 3 is given by
} 
(1997) prove that these conditions imply uniqueness of equilibrium provided that $R=0$ in (11). ${ }^{21}$ Such uniqueness is not necessary or assumed in Proposition 3. In addition, Proposition 3 also covers important cases where $h_{i}$ is not concave. For example; Hirshleifer (1989) proposes the logit specification of the contest success function, with $H=i d$ (the identity function), and $h_{i}\left(s_{i}\right)=e^{k_{i} s_{i}}$ $\left(k_{i}>0\right)$, and studies the special case where $k_{i}=k$ for all $i$ under additional assumptions. In this case, $h_{i}^{\prime \prime}\left(s_{i}\right) / h_{i}^{\prime}\left(s_{i}\right)=k_{i}$. So if, in addition, costs are also exponential, $c_{i}\left(s_{i}\right)=e^{l_{i} s_{i}}$, the conclusions of Proposition 3 continue to apply provided that $k_{i} \leq l_{i}$.

\subsection{Technology Choice in Oligopoly}

As a final application, we consider an important class of games in which oligopoly producers make technology choices (as well as setting output). These games were mentioned bricfly in Section 3.1. Our treatment here will also illustrate how our results with one-dimensional aggregates can be applied when strategy sets are multi-dimensional and also clarifies how "perverse" comparative statics may arise in such games and how it can be ruled out.

Consider a Cournot model with $I$ heterogeneous firms. Let $q=\left(q_{1}, \ldots, q_{I}\right)$ be the output vector and $a=\left(a_{1}, \ldots, a_{I}\right)$ the the technology vector. Let us define $Q=\sum_{j=1}^{I} q_{j}$ as aggregate output. Profit of firm $i$ is

$$
\Pi_{i}\left(q_{i}, a_{i}, Q\right) \equiv \pi_{i}(q, a)=q_{i} P(Q)-c_{i}\left(q_{i}, a_{i}\right)-C_{i}\left(a_{i}\right)
$$

where $P$ is the (decreasing) inverse market demand, $c_{i}$ is the cost of firm $i$ is a function of its

$$
\eta\left(Q^{*}\right) \equiv\left[\frac{2 H^{\prime}\left(H^{-1}\left(Q^{*}\right)\right)}{\left(R+Q^{*}\right)}-\frac{H^{\prime \prime}\left(H^{-1}\left(Q^{*}\right)\right)}{H^{\prime}\left(H^{-1}\left(Q^{*}\right)\right)}\right]^{-1} .
$$

Therefore, when, for example, $H=h_{i}=\mathrm{id}$ (the identity function), and $R=0$, we have $\eta\left(Q^{*}\right)=Q^{*} / 2$, and so player $i$ is "dominant" if and only if $s_{i}^{*} \geq Q^{*} / 2$. In the standard interpretation of a contest, this means that she is dominant when her probability of winning the prize is greater than $1 / 2$-i.e., when she is a favorite in the terminology of Dixit (1987). However, this favorite-to-win interpretation does not necessarily apply for more general games covered by Proposition 3. We therefore use the term "dominant" rather than "favorite".

${ }^{21}$ More recently, Cornes and Hartley (2005) have proposed a very nice and simply proof of this result based on what they refer as "share functions". Although Cornes and Hartley do not consider comparative statics, their "share function" approach could be used to establish results similar to the results in Proposition 3 under these stronger assumptions if, in addition, one also imposed that $R=0$ in (11). $R=0$ amounts to assuming no discounting in patent races and "no wastage" in contests, and is thus quite restrictive.

When $R>0$, the "share function" approach cannot be used to derive robust comparative statics. The reason for this is that the "share function" approach uses the fact that this function is decreasing everywhere, whereas when $R>0$, it may be increasing. To see this, apply the implicit function theorem to the condition $\Psi\left(s_{i}, Q\right)=0$ imposing $h_{2}\left(s_{2}\right)=s_{i}$ for all $i$. Rewrite this in terms of "shares", $z_{\imath}=s_{i} / Q$, so that $\left[-V_{\imath}-(R+Q)^{2} c_{2}^{\prime \prime}\right] d z_{i}=$ $\left[V R Q^{-2}+(R+Q)^{2} c_{i}^{\prime \prime}+c_{i}^{\prime} \cdot Q^{-2}\left(2(R+Q) Q-(R+Q)^{2}\right)\right] d Q$. The coefficient of $d z_{i}$ is clearly negative. When $R=0$, the coefficient of $d Q$ on the right-hand-side is unambigiously positive, hence $d z_{2} / d Q<0$, i.e., agent $i$ 's share function is strictly decreasing. But in general, this may fail when $R>0$ is allowed. In particular, the term $c_{z}^{\prime} \cdot Q^{-2}\left(2(R+Q) Q-(R+Q)^{2}\right)$ will be positive if and only if $Q \geq R$. Clearly, nothing prevents $c_{z}^{\prime}$ from being sufficiently large for this term to dominate so that the share function becomes increasing when $Q<R$. 
quantity and technology choices, and $C_{i}$ is the cost of technology adoption. Assume that $P, c_{i}$ and $C_{i}$ (for each $i$ ) are twice differentiable, $P$ is strictly decreasing decreasing $\left(P^{\prime}(Q)<0\right.$ for all $Q$ ), $C_{i}$ is convex, and $\partial c_{i}\left(q_{i}, a_{i}\right) / \partial q_{\imath} \partial a_{i}<0$ (for each $i$ ), so that greater technology investments reduce the marginal cost of production for each firm.

The first-order necessary conditions for profit maximization are

$$
\begin{aligned}
& \frac{\partial \pi_{i}}{\partial q_{i}}=P^{\prime}(Q) q_{i}+P(Q)-\frac{\partial c_{i}\left(q_{i}, a_{i}\right)}{\partial q_{i}}=0 \\
& \frac{\partial \pi_{i}}{\partial a_{i}}=-\frac{\partial c_{i}\left(q_{i}, a_{i}\right)}{\partial a_{i}}-\frac{\partial C_{i}\left(a_{i}\right)}{\partial a_{i}}=0 .
\end{aligned}
$$

Naturally, we also require the second-order conditions to be satisfied, which here amount to $D_{\left(q_{i}, a_{i}\right)}^{2} \pi_{i}$ being negative semi-definite. Let us now consider the effect of a decline in the cost of technology investment by one of the firms (i.e., a shift in $C_{i}$ ), which clearly corresponds to a positive shock. The results from Section 5 suggest that we should check the local solvability condition. In particular, consider the matrix

$$
D_{\left(q_{i}, a_{i}\right)} \Psi_{i}=\left(\begin{array}{cc}
P^{\prime}(Q)-\frac{\partial^{2} c_{2}}{\partial q_{i}^{2}} & -\frac{\partial^{2} c_{1}}{\partial g_{i} \partial a_{i}} \\
-\frac{\partial^{2} c_{2}}{\partial q_{2} \partial a_{i}} & -\frac{\partial^{2} c_{1}}{\partial a_{i}^{2}}-\frac{\partial^{2} C_{2}}{\partial a_{i}^{2}}
\end{array}\right)
$$

for each $i$. When $c_{i}\left(q_{i}, a_{i}\right)$ is convex, the matrix

$$
\left(\begin{array}{cc}
-\frac{\partial^{2} c_{2}}{\partial q_{2}^{2}} & -\frac{\partial^{2} c_{2}}{\partial q_{2} \partial a_{2}} \\
-\frac{\partial^{2} c_{1}}{\partial q_{2} \partial a_{i}} & -\frac{\partial^{2} c_{2}}{\partial a_{2}^{2}}
\end{array}\right)
$$

is negative semi-definite. Since $P^{\prime}(Q)<0$ and $\partial^{2} C_{i} / \partial a_{i}^{2} \leq 0$, this is sufficient to guarantee that $\left|D \Psi_{i}\right|<0$. Therefore, whenever each $c_{i}\left(q_{i}, a_{i}\right)$ is convex, the local solvability condition is satisfied. Hence, a decline in the cost of technology investments for one of the firms will necessarily increase total output. Similarly, the effects of an increase in demand on output and technology choices can be determined robustly.

Now, the oligopoly-technology game will be a game with strategic substitutes provided merely that $\partial^{2} c_{i}\left(q_{i}, a_{i}\right) / \partial q_{i} \partial a_{i} \leq 0 .^{22}$ So when technological development lowers the marginal cost of producing more input, which of course is perfectly reasonable to assume, the results from Section 4 apply whether or not the local solvability condition is satisfied (and whether or not payoff functions are pseudo-concave in own strategies, etc.). In particular, an equilibrium will exist, and any positive shock to an individual player will lead to an increase in that player's equilibrium strategies $\left(q_{i}^{*}, a_{i}^{*}\right)$ and a decrease in the aggregate of the opponents' strategies $\sum_{j \neq i} q_{j}^{*}$ (note here

\footnotetext{
${ }^{22}$ This condition ensures that payoff functions are supermodular in own strategies. It is easy to check that payoff functions also exhibit decreasing differences in own and opponents' strategies.
} 
that the aggregator is one-dimensional, i.e., $g(s)=\sum_{j} q_{j}$, so the statement on the aggregate of the opponents' strategies concerns only quantities). The effect of shocks to the aggregate is determined also (Theorem 3) and so are the effect of entry (Theorem 4) and the impact on payoffs (Corollary 1).

We next provide a specific example with strategic substitutes where the local solvability condition is violated. As will become clear, this leads to "perverse" comparative statics conclusions in the sense that the conclusion of Theorem 7 is violated. Concretely, a positive shock to a player will be seen to imply a decrease in the aggregate. But we repeat that the above mentioned conclusions on, for example, individual shocks' effect on individual strategies remain valid.

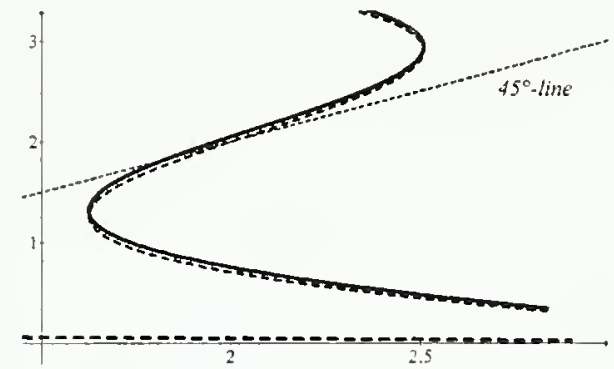

Figure 1: Backward reply correspondences Figure 2: A decrease in marginal costs for the (dashed). Aggregate backward reply correspon- second firm leads to a decrease in aggregate outdence (solid).

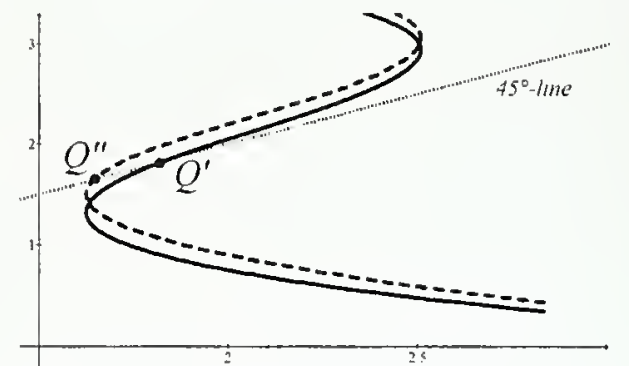
put from $Q^{\prime}$ to $Q^{\prime \prime}$.

Suppose that there are only two firms and $P(Q)=K-Q$ for some constant $K>0$. Suppose also that firm 1's costs are given by $0.5 q_{1}^{2}\left(\alpha_{1}-a_{1}\right)+C_{1}\left(a_{1}\right)$ for some differentiable, strictly increasing and strictly convex function $C_{1}$. This implies that its payoff function is

$$
\pi_{i}(q, a)=[K-Q] q_{1}-0.5 q_{1}^{2}\left(\alpha_{1}-a_{1}\right)-C_{1}\left(a_{1}\right)
$$

The first-order conditions for firm 1 can be written as,

$$
K-Q-q_{1}-q_{1}\left(\alpha_{1}-a_{1}\right)=0 \text {, and } 0.5 q_{1}^{2}=C_{1}^{\prime}\left(a_{1}\right)
$$

Since $C_{1}$ is strictly increasing, $\left(C_{1}^{\prime}\right)^{-1}$ is well-defined. $\left(C_{1}^{\prime}\right)^{-1}$ is strictly increasing since $C_{1}$ is strictly convex (and conversely, when $\left(C_{1}^{\prime}\right)^{-1}$ is strictly increasing, $C_{1}$ must be strictly convex). Let us define $G_{1}(z) \equiv\left(C_{1}^{\prime}\right)^{-1}\left(0.5 z^{2}\right)$ which will also be strictly increasing. Choosing $G_{1}$ is equivalent 
to choosing $C_{1}$. Let

$$
G_{1}\left(q_{1}\right)=-\delta_{1} q_{1}^{2}+\gamma_{1} q_{1}+\beta_{1}
$$

where $\gamma_{1}, \delta_{1}>0$, and $\beta_{1}<2$, so that the best responds choice of quantity for firm 1 becomes the solution to the following cubic equation:

$$
K-Q+\left(\beta_{1}-1-\alpha_{1}\right) q_{1}-\delta_{1} q_{1}^{3}+\gamma_{1} q_{1}^{2}=0
$$

Figure 1 plots $q_{1}$ as a function of $Q$ for a particular choice of parameters (the dashes "inverse- $S$ " shaped curve). The second dashed curve (the negatively sloped line) shows the same relationship for firm $2\left(q_{2}\right.$ as a function of $\left.Q\right)$. Concretely, firm 2's cost function is assumed to take the form: $\left[\alpha_{2}+0.5 \beta_{2} q_{2}^{2}-\gamma_{2}\left(a_{2}\right)^{1 / 2}\right]+\delta_{2} a_{2}$. This yields a simple linear relationship between $Q$ and $q_{2}$ : $0=K-Q-\beta_{2} q_{2}$. The solid line in the figure is the aggregate backward reply correspondence which shows $q_{1}+q_{2}$ as a function of $Q$ (the sum of the two dashed curves). ${ }^{23}$

A Cournot equilibrium is given by the solid curve's intersection with the $45^{\circ}$-line in Figure 1. Figure 2 depicts the same aggregate backward reply correspondence as in Figure 1 (solid), together with a similarly constructed aggregate backward reply correspondence (dashed). The only difference between the two's parameter values is that for the dashed curve $\beta_{2}$ is lower. ${ }^{24}$ Naturally, a reduction in $\beta_{2}$ corresponds to a reduction in the marginal cost of firm 2 . The figure shows that such a decrease in marginal costs reduces aggregate output $Q$. It can also be verified for the parameters here, the two firms' payoff/profit functions are strictly concave (even though the cost function of firm 1 is not convex). This example thus illustrates that even in "nice" aggregative games with strategic substitutes, the local solvability condition is critical for the conclusion of Theorem 7: Unless the shock hits the aggregate (e.g., an increase in $K$ ) so that Theorem 3 applies, a positive shock may lead to a decrease in the equilibrium aggregate when the local solvability condition does not hold.

\section{Multidimensional Aggregates}

We have so far focused on aggregative games with one-dimensional aggregates, i.e., games where $g: S \rightarrow \mathbb{R}$. Many important examples, including the Bertrand game with homogeneous products discussed in Section 3, require more than a one-dimensional aggregate, $g: S \rightarrow \mathbb{R}^{M}, M>1$. Another game with multi-dimensional aggregates is the technology choice game considered in

\footnotetext{
${ }^{23}$ The specific set of parameter values yielding the configuration in Figure 1 are: $K=4, \beta_{1}-1-\alpha_{1}=-4.4$, $\gamma_{1}=2.5, \delta_{1}=0.4$, and $\beta_{2}=40$. Note that given these parameter values $G_{1}$ will be strictly increasing $\left(C_{1}\right.$ will be strictly convex) whenever $q_{1}<3.125$. It is also straightforward to verify that any perturbation of these parameters leads to the same comparative static results, so that this perverse comparative static is "robust".

${ }^{24}$ Concretely, $\beta_{2}=10$ for the dashed curve and $\beta_{2}=40$ for the solid curve.
} 
Section 6.3 when technology costs also depend on some aggregate of the technology choices of other firms, e.g., $C_{i}=C_{i}\left(a_{i}, A\right)$ for some aggregate of technology choices $A$.

In this section, we discuss how some of our results can be extended to this case. To simplify the exposition we focus on the case where the aggregator takes the form $g(s)=\sum_{j=1}^{I} s_{j}$. ${ }^{25}$ In this case, naturally, $g: S \rightarrow \mathbb{R}^{N}$, hence $M=N$. We continue to assume that there are $I$ players and we denote the set of players by $\mathcal{I}$. In addition, we assume that the game is both "nice" (Definition 6) and also exhibits strategic substitutes. Then, proceeding as in Section 5, we define $D_{1} \Pi_{i}\left(s_{i}, Q, t\right) \equiv D_{s_{i}} \Pi_{i}\left(s_{i}, Q, t\right)$ and $D_{2} \Pi_{i}\left(s_{i}, Q, t\right) \equiv D_{Q} \Pi_{i}\left(s_{i}, Q, t\right)$. The marginal payoff for player $i$ can again then be expressed as:

$$
D_{s_{i}} \pi_{i}(s, t) \equiv D_{1} \Pi_{i}\left(s_{i}, \sum_{j=1}^{I} s_{j}, t\right)+D_{2} \Pi_{i}\left(s_{i}, \sum_{j=1}^{I} s_{j}, t\right) .
$$

Now denoting the vector of aggregates by $Q \equiv \sum_{j=1}^{I} s_{j}$, we again define:

$$
\Psi_{i}\left(s_{i}, Q, t\right) \equiv D_{1} \Pi_{i}\left(s_{i}, Q, t\right)+D_{2} \Pi_{i}\left(s_{i}, Q, t\right) .
$$

Parallel with the local solvability condition (Definition 7 in Section 5), we will place certain key restrictions on the $\Psi_{i}$ functions. These restrictions, together with our focus on nice games with strategic substitutes, are collected in the following assumption.

Assumption 1 The game $\Gamma$ is an aggregative nice game (Definition 6) and in addition, for each player $i$, we have:

- (Strategic Substitutes) $S_{i}$ is a compact lattice, and $\pi_{i}\left(s_{i}, s_{-i}, t\right)$ is supermodular in $s_{i}$ and exhibits decreasing differences in $s_{i}$ and $s_{j}$ (for all $j \neq i$ ).

- (Strong Local Solvability) Every real eigenvalue of $D_{s_{i}} \Psi_{i}\left(s_{i}, \sum_{j=1}^{I} s_{j}, t\right)$ is negative.

Remark 7 (Strong Local Solvability) That every real eigenvalue of $D_{s_{2}} \Psi_{i}\left(s_{i}, \sum_{j=1}^{I} s_{j}, t\right)$ is negative implies that its determinant is non-zero (this is because $D_{s_{i}} \Psi_{i}$ must have non-negative offdiagonal elements, see the proof of Theorem 11 for further details). Consequently, local solvability (Definition 7) is implied by strong local solvability.

Assumption 1 is straightforward to verify because of the following two relationships linking the usual second-order matrices of $\pi_{i}$ and the gradient of the $\Psi_{i}$ functions:

\footnotetext{
${ }^{25}$ All of the following results remain valid if we assume instead that $g(s)=\left(g^{1}\left(s_{1}^{1}, \ldots, s_{I}^{1}\right), \ldots, g^{N}\left(s_{1}^{N}, \ldots, s_{I}^{N}\right)\right)$ with each function $g^{n}$ separable. See the beginning of the proof of Theorem 7 for details on how one can transform such a game into a game with a linear aggregator.
} 


$$
\begin{array}{r}
D_{s_{i} s_{j}}^{2} \pi_{i}(s, t) \equiv D_{s_{i} s_{j}}^{2} \Pi_{i}\left(s_{i}, \sum_{k=1}^{I} s_{k}, t\right) \equiv D_{Q} \Psi_{i}\left(s_{i}, \sum_{k=1}^{I} s_{k}, t\right) \text { for all } j \neq i, \text { and } \\
D_{s_{i} s_{i}}^{2} \pi_{i}(s, t) \equiv D_{s_{i} s_{2}}^{2} \Pi_{i}\left(s_{i}, \sum_{j=1}^{I} s_{j}, t\right) \equiv D_{s_{i}} \Psi_{i}\left(s_{i}, \sum_{j=1}^{I} s_{j}, t\right)+D_{Q} \Psi_{i}\left(s_{i}, \sum_{j=1}^{I} s_{j}, t\right) .
\end{array}
$$

Since by (15), $D_{Q} \Psi_{i} \equiv D_{s_{i} s_{\jmath}}^{2} \Pi_{i}$ for all $j \neq i$, decreasing differences (strategic substitutes) requires simply that $D_{Q} \Pi_{i}\left(s_{i}, \sum_{j=1}^{I} s_{j}, t\right)$ is a non-positive matrix. Next we can sum the two matrices $D_{s_{i}} \Psi_{i}$ and $D_{Q} \Psi_{i}$ in order to obtain $D_{s_{i} s_{2}}^{2} \pi_{i}$ (cf. (15)). Supermodularity holds if and only if the matrix $D_{\boldsymbol{s}_{\imath} s_{i}}^{2} \Pi_{i}$ has non-negative off-diagonal entries. Finally, strong local solvability requires that the real eigenvalues of $D_{s_{2}} \Psi_{i}$ are negative. When $D_{s_{2}} \Psi_{i}$ is symmetric (which is often the case in practice), this is the same as $D_{s_{i}} \Psi_{i}$ being a negative definite matrix. Note also that concavity of payoff functions in own strategies is implied by Assumption 1 (see the proof of Theorem 11). Thus, in games with multi-dimensional aggregates the verification of strong local solvability "replaces" the very similar task of verifying that the Hessian is negative definite. The concavity implications of Assumption 1 also mean that when this assumption holds, the existence of a pure-strategy Nash equilibrium follows immediately by Brouwer's fixed point theorem. This is noted in the following theorem (proof omitted):

Theorem 10 (Existence) Suppose that $\Gamma$ satisfies Assumption 1. Then $\Gamma$ has a (pure-strategy) Nash equilibrium.

We next define the backward reply function of player $i$ again using the first-order conditions: $s_{\imath}=b_{i}(Q, t) \Leftrightarrow \Psi_{i}\left(s_{i}, Q\right)=0$. Assumption 1 simplifies matters here by ensuring that each $Q$ leads to a unique backward reply function (rather than a correspondence), $b_{i}(Q, t){ }^{26}$ For any given vector of aggregates $Q$, the gradient of $b_{i}(Q)$ is also well-defined and is given by:

$$
D_{Q} \dot{b}_{i}(Q, t)=-\left[D_{s_{i}} \Psi_{i}\left(b_{i}(Q, t), Q, t\right)\right]^{-1} D_{Q} \Psi_{i}\left(b_{i}(Q, t), Q, t\right)
$$

and thus

$$
D_{Q} b(Q, t)=\sum_{j=1}^{I} D_{Q} b_{j}(Q, t) .
$$

\footnotetext{
${ }^{26}$ Fixing $Q$, it is clear that the gradient of $\Psi_{2}(\cdot, Q), D_{s_{2}} \Psi_{i}\left(s_{2}, Q\right)$ (which is a $N \times N$ matrix), is non-singular at any stationary point. In particular, from strong local solvability, the determinant of $D_{s_{2}} \Psi_{2}\left(s_{2}, Q\right)$ never changes sign and never equals zero. This immediately implies that there exists a unique critical point (e.g., from the Poincare-Hopf theorem; Milnor (1965)).
} 
Let us also recall that an $M$-matrix is a matrix with positive real eigenvalues and non-positive off-diagonal entries. ${ }^{27}$ We are then ready to state the following multi-dimensional version of Theorems 3 and 7 .

Theorem 11 (Shocks to the Aggregates) Suppose that $\Gamma$ satisfies Assumption 1. Let $t \in T \subseteq$ $\mathbb{R}^{N}$ be a shock to the aggregate, that is, let $\pi_{i}(s, t) \equiv \Pi_{i}\left(s_{i}, t+\sum_{j=1}^{I} s_{j}\right)$ for all $i \in \mathcal{I}$, and assume that the matrix $\mathbf{I}-\left[D_{Q} b(Q+t)\right]^{-1}$ exists and is non-singular. Then:

- (Sufficency) If the matrix $\mathbf{I}-\left[D_{Q} b(Q+t)\right]^{-1}$ is an $M$-matrix (for all $Q$ and $t$ ), an increase in $t \in T$ leads to a decrease in each component of the equilibrium aggregate vector.

- (Necessity) Conversely, let $Q\left(t^{\prime}\right)$ be an equilibrium aggregate given some vector of parameters $t^{\prime} \in T$ that hits the aggregate. Then if $\mathbf{I}-\left[D_{Q} b\left(Q\left(t^{\prime}\right)+t^{\prime}\right)\right]^{-1}$ is not an $M$-matrix, there exists $t^{\prime \prime}>t^{\prime}$ such that at least one component of the equilibrium aggregate vector increases when $t$ is raised from $t^{\prime}$ to $t^{\prime \prime}$.

\section{Proof. See Section 11.7.}

In what follows, we will use the sufficiency part of Theorem 11 to present direct parallels to the other theorems presented in Section 4. Nevertheless, the necessity part of this theorem is also noteworthy, perhaps even surprising.

Given Theorem 11, the proofs of the next three theorems closely follow the proofs of the analogous theorems for the one-dimensional case and are thus are omitted. ${ }^{28}$ For the next theorem, suppose that the default inaction strategy of the entrant now is a vector of zeroes (or, more generally, the least element in the entrant's strategy set).

Theorem 12 (Entry) Suppose that $\Gamma$ satisfies Assumption 1 and the sufficiency conditions in Theorem 11. Then entry of an additional player leads to a decrease in the aggregates of the existing players. In addition, at least one of the aggregates of all players must increase with entry, and strictly so unless the entrant chooses inaction.

Theorem 13 (Idiosyncratic Shocks) Suppose that $\Gamma$ satisfies Assumption 1 and the sufficiency conditions in Theorem 11. Then a positive idiosyncratic shock to player $i \in \mathcal{I}$ leads to an increase

\footnotetext{
${ }^{27}$ Recall that an $M$-matrix and an inverse $M$-matrix are also $P$-matrices (i.e., all of their principal minors are positive). Moreover, if a matrix has a non-positive off-diagonal, it is an $M$-matrix if and only if it is also a $P$-matrix.

${ }^{28}$ The only new feature is the second statement of the entry theorem (that at least one of the aggregates must increase upon entry). This is a direct consequence of the fact that the backward reply function of the existing players, $\bar{b}$, is decreasing (this is proved as part of Theorem 11). Indeed, let $Q^{b}$ be the vector of aggregates before entry, $Q^{\alpha}$ the aggregates after entry, and $s_{I+1} \geq 0$ be the strategy chosen by the entrant. Since $\bar{b}$ is decreasing, $Q^{a} \leq Q^{b}$ implies: $0 \leq Q^{b}-Q^{a}=\bar{b}\left(Q^{b}\right)-\bar{b}\left(Q^{a}\right)-s_{I+1} \leq-s_{I+1}$ which in turn implies $s_{I+1}=Q^{b}-Q^{a}=0$.
} 
in this player's equilibrium strategy and to a decrease in the associated aggregates of the existing players.

Remark 8 (Sufficient Conditions for Two-Dimensional Aggregates) When $N=2$ ( $g$ : $\left.S \rightarrow \mathbb{R}^{2}\right)$, the sufficient conditions are particularly easy to verify. In particular, I- $\left[D_{Q} b(Q+t)\right]^{-1}$ exists and is a non-singular $M$-matrix when $-\left[D_{Q} b(Q+t)\right]^{-1}$ is a non-singular $M$-matrix. This is generally the case (regardless of $N$ ), since the real eigenvalues of $\left[\mathbf{I}-\left[D_{Q} b(Q+t)\right]^{-1}\right]$ are equal to $\left(\lambda_{1}+1\right), \ldots,\left(\lambda_{M}+1\right)$, where $\lambda_{1}, \ldots, \lambda_{M}>0$ are the real eigenvalues of $-\left[D_{Q} b(Q+t)\right]^{-1}$.

In this two-dimensional case, $-\left[D_{Q} b(Q+t)\right]^{-1}$ is a non-singular $M$-matrix if and only if the determinant of $D_{Q} b(Q+t)$ is positive. To see this first note that since $D_{Q} b(Q+t)$ is a nonpositive matrix, its trace is non-positive. So when the determinant is positive, both eigenvalues must be negative (when they are real; if they are not real, then there is nothing to check because the definition of an $M$-matrix above requires only that the real eigenvalues be positive). It then follows that $-\left[D_{Q} b(Q+t)\right]^{-1}$ is a matrix with non-positive off-diagonal elements and positive (real) eigenvalues, and thus it is a non-singular $M$-matrix.

Now since $D_{Q} b(Q+t)=\sum_{j=1}^{I} D_{Q} b_{j}(Q+t)$, a sufficient condition for $D_{Q} b(Q+t)$ to have a positive determinant is that each of the matrices $D_{Q} b_{\imath}(Q+t), i=1, \ldots, I$ is quasi-negative definite $\left(x^{T} D_{Q} b_{i}(Q+t) x<0\right.$ for all $\left.x \neq 0\right)$. This is because the sum of quasi-negative definite matrices is quasi-negative definite, and a $2 \times 2$ quasi-negative definite matrix has a positive determinant. The next corollary exploits this observation.

Corollary 2 (Symmetric Games with Two-Dimensional Aggregates) Suppose that $\Gamma$ satisfies Assumption 1 and $N=2$. Consider a shock to the aggregate. Then if the matrix $D_{Q} \Psi_{i}\left(s_{i}, \sum_{j=1}^{I} s_{j}+t\right)$ has a positive determinant for all $s \in S$ and $t \in T$, a positive shock to the aggregates will lead to a decrease in both of the aggregates in any symmetric equilibrium. In addition, the results in Theorems 12-13 continue to hold when the existing players choose identical strategies before and after entry (Theorem 12), and the players that are not affected by the shock choose identical strategies before and after the arrival of the idiosyncratic shock (Theorem 13).

Proof. The aggregate in a symmetric equilibrium is given by $Q=I b_{i}(Q+t)$ where $i \in I$ is any of the (identical) players. From Theorem 11, a positive shock to the aggregate decreases the aggregate if only if $\left[\mathbf{I}-\left[D_{Q} b(Q+t)\right]^{-1}\right.$ is a non-singular $M$-matrix. From Remark 8 , we only need to verify that $-\left[D_{Q} b(Q+t)\right]^{-1}=-\left[D_{Q} b_{l}(Q+t)\right]^{-1} / I$ is a non-singular $M$-matrix. When $N=2$, this holds if and only if the determinant of $D_{Q} b_{i}(Q+t)$ is positive. This is the case when $D_{Q} \Psi_{i}$ has a positive determinant, because $D_{s_{i}} \Psi_{i}$ has a positive determinant and $D b_{i}=-\left[D_{s_{2}} \Psi_{\imath}\right]^{-1} D_{Q} \Psi_{i}$. 


\section{Nice Games without Differentiability}

In this section, we extend the results for nice games presented in Section 5 . Recall that the main assumption of Section 5, local solvability, presupposes that payoff functions and the aggregator are twice continuously differentiable. In this section, we show that robust comparative statics can be derived without differentiability as long as a non-differentiable version of the local solvability condition is imposed. We limit attention to the case of one-dimensional strategy sets (hence the aggregate must be one-dimensional also). Recall that an aggregator always has a representation of the form $g(s)=H\left(\sum_{j=1}^{I} h_{j}\left(s_{j}\right)\right)$, where $H$ and $h_{1}, \ldots, h_{i}$ are strictly increasing functions. Therefore, for any $Q$ in the range of $g$, we have $Q=g(s) \Leftrightarrow s_{i}=h_{i}^{-1}\left[H^{-1}(Q)-\sum_{j \neq i} h_{j}\left(s_{j}\right)\right]$. Intuitively, this means that if we know the aggregate $Q$ and the strategies of $I-1$ players, we also know the strategy of the last player. Let us also define $G_{i}(Q, y) \equiv h_{i}^{-1}\left[H^{-1}(Q)-y\right]$. Recall from Milgrom and Shannon (1994) that a function $f(Q, y)$ satisfies the single-crossing property in $(Q, y)$ if, for all $Q^{\prime}>Q$ and $y^{\prime}>y$, we have

$$
f\left(Q^{\prime}, y\right) \geq(>) f(Q, y) \Rightarrow f\left(Q^{\prime}, y^{\prime}\right) \geq(>) f\left(Q, y^{\prime}\right)
$$

The main result in this section, presented next, shows that an appropriately-chosen singlecrossing property can replace the local solvability condition (Definition 7) and thus extends our results to nice games without differentiability.

Theorem 14 (Comparative Statics for Nice Games without Differentiability) Consider an aggregative game with one-dimensional convex, compact strategy sets, a separable aggregator, payoff functions that are upper semi-continuous and quasi-concave in own strategies. Suppose that $\Pi_{i}\left(G_{i}(Q, y), Q, t\right)$ (for each $i \in \mathcal{I}$ ) satisfies the single-crossing property in $(Q, y)$. Then the conclusions of Theorems 7 and 8 continue to hold. Moreover, provided that payoff functions are twice differentiable and the equilibrium is interior, the conclusions of Theorem 9 also hold.

Proof. See Section 11.8 .

Notice that differentiability is needed in Theorem 9 in order to even state this theorem's main conclusions. Clearly, the more interesting part of Theorem 14 concerns Theorems 7-8.

The next corollary uses the insights of Theorem 14 to provide another useful and simple alternative to the local solvability condition for nice games.

Corollary 3 Consider a nice aggregative game with linear aggregator $g(s)=\sum_{i} s_{i}$, one-dimensional strategy sets (i.e., $N=1$ ), and assume that for each player $i$ :

$$
D_{s_{i}} \Psi_{i}\left(s_{i}, Q\right) \leq 0 \text { for all } s_{i} \text { and } Q
$$


Then the conclusions of Theorems 7,8 , and 9 continue to hold.

Proof. Since $g$ is linear, $G_{i}(Q, y)=Q-y$ and $\Pi_{i}\left(G_{i}(Q, y), Q, t\right)=\Pi_{i}(Q-y, Q, t)$ (for each $i \in \mathcal{I})$. The condition $D_{s_{1}} \Psi_{i}\left(s_{i}, Q\right) \leq 0$ is equivalent to $-D_{s_{i}} \Psi_{i}=-D_{11}^{2} \Pi_{i}-D_{21}^{2} \Pi_{i} \geq 0$ for all $s_{i}$ and $Q$. This is in turn equivalent to $\prod_{i}(Q-y, Q, t)$ exhibiting increasing differences in $Q$ and $y$. Since increasing differences implies the single-crossing property, the results follow from Theorem 14.

Note that Condition (17) in Corollary 3 requires that $D_{s_{2}} \Psi_{i}\left(s_{i}, Q\right) \leq 0$ for all $s_{i}$ and $Q$. By contrast, the local solvability condition requires $D_{s_{i}} \Psi_{i}\left(s_{i}, Q\right) \neq 0$, but only when $s_{i}$ and $Q$ are such that $\Psi_{i}\left(s_{i}, Q\right)=0$. Thus neither condition generalizes the other. If (17) holds with strict inequality throughout, i.e., $D_{s_{i}} \Psi_{i}\left(s_{i}, Q\right)<0$ for all $s_{i}$ and $Q$, then local solvability would be implied, though the weak inequality makes this condition easier to check and apply in a variety of examples (recall the discussion in Section 6.1). ${ }^{29}$

\section{Walrasian Play}

When the aggregate $Q$ results from the "average" of the strategies of a large number of players, it may plausible to presume that each player $i \in \mathcal{I}$ will ignore the effect of its strategy on the aggregate. In this case, each player $i \in \mathcal{I}$ will maximize the reduced payoff function $\Pi_{i}=$ $\Pi_{i}\left(s_{i}, Q, t\right)$ with respect to $s_{i}$ taking $Q$ as given. This is the behavior assumed in standard general equilibrium theory with a finite (but large) number of households. With analogy, we refer to the situation in aggregative games where players ignore their impact on aggregates as Walrasian play and the associated Nash equilibrium as a Walrasian Nash Equilibrium. ${ }^{30}$ For such games, our results can be strengthened (and the proofs in fact become nore straightforward). Here we briefly outline the main results in this case, focusing on one-dimensional aggregates (i.e., $K=1$ ).

Definition 10 (Walrasian Nash Equilibrium) Consider an aggregative game $\Gamma=\left(\left(\Pi_{2}, S_{2}\right)_{2} \in \mathcal{I}, g, T\right)$. The strategy profile $s^{*}(t)=\left(s_{1}^{*}(t), \ldots, s_{I}^{*}(t)\right)$ is called a (pure-strategy) Walrasian Nash equilibrium given $t \in T$ if holding $Q(t)=g\left(s^{*}(t)\right)$ fixed, we have for each player $i=1, \ldots, I$ that,

$$
s_{i}^{*}(t) \in R_{i}(Q, t) \equiv \arg \max _{s_{i} \in S_{2}} \Pi_{i}\left(s_{i}, Q(t), t\right) .
$$

\footnotetext{
${ }^{29}$ It can also be noted that (17) with strict inequality makes up "half" of what Corchón (1994) calls the "strong concavity" condition. The other "half" of Corchon's strong concavity condition requires payoff functions to exhibit strictly decreasing differences in own and opponents' strategies. This is not assumed in our analysis.

${ }^{30}$ Such "aggregate-taking" behavior has been studied extensively within evolutionary game theory, see for example Vega-Redondo (1997), Possajennikov (2003), and Schipper (2004).
} 
Notice that under Walrasian play, a player's best responses $R_{i}(Q, t)$ will depend on the aggregate $Q$ and the exogenous variables $t$. An increase in $t$. is a positive shock for player $i$ if the smallest and largest selections from $R_{i}(Q, t)$ are both increasing in $t$. The game features strategic substitutes if each $S_{i}$ is a lattice, $\Pi_{i}$ is supermodular in $s_{i}$, and exhibits decreasing differences in $s_{i}$ and $Q$. When $N=1$ and $\Pi_{i}$ is twice continuously differentiable, a sufficient condition for $t$ to be a positive shock is that $D_{s_{i}}^{2} \Pi_{i}\left(s_{i}, Q, t\right) \geq 0$ (for all $Q$ and $t$ ), and a sufficient condition for strategic substitutes is that $D_{s_{i}}^{2} \Pi_{i}\left(s_{i}, Q, t\right) \leq 0$ (for all $Q$ and $t$ ).

As in previous sections, we maintain the compactness and upper semi-continuity assumptions. Also, since as before, there may be multiple equilibria, we continue to focus on the smallest and largest equilibrium aggregates $Q_{*}(t)$ and $Q^{*}(t)$ (cf. equations (6) and (5) in Section 4).

Theorem 15 (Comparative Statics for Walrasian Nash Equilibria) Consider an aggregative $\Gamma$ and assume that the (reduced) payoff function $\Pi_{i}\left(s_{i}, Q, t\right)$ is quasi-concave in $s_{i} \in S_{i}$ for each $i \in \mathcal{I}$. Then a Walrasian Nash equilibrium exists. Moreover, we have that:

1. Theorems 7, 8, and 9 hold for Walrasian Nash equilibria. In particular, a positive shock to one or more of the agents will lead to an increase in the smallest and largest equilibrium aggregates, and entry increases the smallest and largest equilibrium aggregates. In addition, suppose that payoff functions are smooth and the equilibrium is interior. Then for each $i \in \mathcal{I}, s_{i}^{*}(t)$ is locally coordinatewise increasing in a positive shock $t$ provided that:

$$
-\left[D_{s_{2} s_{\imath}}^{2} \Pi_{i}\left(s_{i}^{*}(t), Q(t), t\right)\right]^{-1} D_{s_{1} Q}^{2} \Pi_{i}\left(s_{i}^{*}(t), Q(t), t\right) \geq 0
$$

and if $\Pi_{i}=\Pi_{i}\left(s_{i}, Q\right)$ (i.e., the shock does not directly affect player $\left.i\right)$, then the sign each element of the vector $D_{t} s_{i}^{*}(t)$ is equal to the sign of each element of the vector

$$
-\left[D_{s_{i} s_{i}}^{2} \Pi_{i}\left(s_{i}^{*}(t), Q(t)\right)\right]^{-1} D_{s_{i} Q}^{2} \Pi_{i}\left(s_{i}^{*}(t), Q(t)\right) .
$$

2. If $\Gamma$ features strategic substitutes, then Theorems 3, 4, and 5 continue to hold for Walrasian Nash equilibria.

Proof. (Sketch) For part 1, simply define $Z(Q, t) \equiv\left\{g(s) \in \mathbb{R}: s_{i} \in R_{i}(Q, t)\right.$ for all $\left.i\right\}$. In view of our compactness and upper semi-continuity assumptions, $Z(Q, t)$ is a convex-valued, upper hemi-continuous correspondence. Then we can proceed as in the proof of Theorem 7 but using $Z(Q, t)$ instead of the function $\bar{q}$. Figure 10 in the Appendix makes it clear that the general argument remains valid if instead of the function $\bar{q}$, we use a convex-valued correspondence. Given this result, the proofs of Theorems 8 , and 9 apply with minimal modifications. 
For part 2, note that a shock to the aggregate is a negative shock (by decreasing differences in $s_{i}$ and $Q$ ), hence it leads to a decrease in the smallest and largest aggregates by the conclusion from part 1. The conclusions of Theorems 4 and 5 are established by straightforward modifications of the original proofs.

A noteworthy implication of Theorem 15 is that all our results for nice aggregate games continue hold for a Walrasian Nash equilibria without imposing local solvability or differentiability and boundary conditions (only quasi-concavity is imposed to ensure that best response correspondences are convex-valued). This highlights that the challenge in deriving robust comparative static results in aggregative games lies in limiting the magnitude of the effect of own strategies on the aggregate. It should also be noted that part 2 of the theorem is false if payoff functions are not assumed to be quasi-concave, though the results do hold if the game instead features strict strategic substitutes ( $i$.e., if strictly decreasing differences is assumed instead of decreasing differences in Definition 3). ${ }^{31}$ The proof of Theorem 15 also shows that any separability assumptions on the aggregator $g$ are unnecessary: the conclusions hold provided that $g$ is an increasing function (without further restrictions).

\section{Conclusion}

This paper presented robust comparative static results for aggregative games and showed how these results can be applied in several diverse settings. In aggregative games, each player's payoff depends on her own actions and on an aggregate of the actions of all players (for example, sum, product or some moment of the distribution of actions). Many common games in industrial organization, political economy, public economics, and macroeconomics can be cast as aggregative games. Our results focused on the effects of changes in various parameters on the aggregates of the game. In most of these situations the behavior of the aggregate is of interest both directly and also indirectly, because the comparative statics of the actions of each player can be obtained as a function of the aggregate. For example, in the context of a Cournot model, our results characterize the behavior of aggregate output, and given the response of the aggregate to a shock, one can then characterize the response of the output of each firm in the industry.

We focused on two classes of aggregative games: (1) aggregative of games with strategic substitutes and (2) "nice" aggregative games, where payoff functions are twice continuously differentiable, and (pseudo-)concave in own strategies. For example, for aggregative games with strategic substitutes, we showed that:

\footnotetext{
${ }^{31}$ However, an equilibrium is not guaranteed to exist in this case because of lack of quasi-concavity. To apply the result mentioned in the text one must thus first (directly) establish the existence of an equilibrium.
} 
1. Changes in parameters that only affect the aggregate always lead to an increase in the aggregate (in the sense that the smallest and the largest elements of the set of equilibrium aggregates increase).

2. Entry of an additional player decreases the (appropriately-defined) aggregate of the strategies of existing players.

3. A "positive" idiosyncratic shock, defined as a parameter change that increases the marginal payoff of a single player, leads to an increase in that player's strategy and a decrease in the aggregate of other players' strategies.

We provided parallel, and somewhat stronger, results for nice games under a local solvability condition (and showed that such results do not necessarily apply without this local solvability condition).

The framework developed in this paper can be applied to a variety of settings to obtain "robust" comparative static results that hold without specific parametric assumptions. In such applications, our approach often allows considerable strengthening of existing results and also clarifies the role of various assumptions used in previous analyses. We illustrated how these results can be applied and yield sharp results using several examples, including public good provision games, contests, and oligopoly games with technology choice.

Our results on games with multi-dimensional aggregates (Section 7) are only a first step in this direction and our approach in this paper can be used to obtain additional characterization results for such games. We leave a more systematic study of games with multi-dimensional aggregates to future work. We also conjecture that the results presented in this paper can be generalized to games with infinitely many players and with infinite-dimensional strategy sets. In particular, with the appropriate definition of a general aggregator for a game with infinitely many players (e.g., along the lines of the separability definitions in Vind and Grodal (2003), Ch. 12-13), our main results and in fact even our proofs remain valid in this case. Similarly, with the appropriate local solvability condition in infinite dimension, all of our results also appear to generalize to games with infinite-dimensional strategy sets. The extension of these results to infinite-dimensional games is another area for future work. 


\section{Appendix: Proofs}

\subsection{Proof of Theorem 2}

For each player $i$, define the correspondence $\operatorname{Gr}\left[R_{i}\right]: T \rightarrow 2^{S}$ by,

$$
\operatorname{Gr}\left[R_{i}\right](t) \equiv\left\{s \in S: s_{i} \in R_{i}\left(s_{-i}, t\right)\right\}, t \in T
$$

This correspondence is upper hemi-continuous and has a closed graph: if $s_{i}^{m} \in R_{\imath}\left(s_{-i}^{m}, t^{m}\right)$ for a convergent sequence $\left(s^{m}, t^{m}\right) \rightarrow(s, t)$, then by the fact that $R_{\imath}$ itself has a closed graph, $s_{i} \in R_{i}\left(s_{-\imath}, t\right)$. Moreover, $E(t)=\cap_{i} \operatorname{Gr}\left[R_{i}^{t}\right]$. The correspondence $E: T \rightarrow 2^{S}$ is thus given by the intersection of a finite number of upper hemi-continuous correspondences, and so is itself upper hemi-continuous. In particular, $E$ has compact values $(E(t) \subseteq S$, where $S$ is compact). Therefore, the existence of the smallest and largest equilibrium aggregates, $Q_{*}(t)$ and $Q^{*}(t)$, follows from the continuity of $g$ and from Weierstrass' theorem. Upper semi-continuity of $Q^{*}: T \rightarrow \mathbb{R}$ follows directly from the fact that $g$ is upper semi-continuous and $E$ is upper hemi-continuous (see Ausubel and Deneckere (1993), Theorem 1). Lower semi-continuity of $Q_{*}$ follows by the same argument since $Q_{*}(t) \equiv-\max _{s \in E(t)}-g(s)$ and $g$ is also lower semi-continuous. Finally, when the equilibrium aggregate is unique for all $t, Q_{*}(t)=Q^{*}(t)$ and so is both upper and lower semi-continuous and thus continuous in $t$ on $T$.

\subsection{Proof of Theorem 3}

Recall that $\pi_{i}(s, t) \equiv \Pi_{i}\left(s_{i}, G(g(s), t)\right)$ all $i$, where $g: S \rightarrow \mathbb{R}$ is separable in $s$ and $G(g(s), t)$ is separable in $(s, t)$. This implies that,

$$
G(g(s), t)=H\left(h_{T}(t)+\sum_{j=1}^{I} h_{j}\left(s_{j}\right)\right) .
$$

where $g(s)=M\left(\sum_{i \in \mathcal{I}} h_{i}\left(s_{i}\right)\right)$ is the aggregator of Definition 1, and $M$ is a strictly increasing function. Moreover, recall that best response correspondence $R_{i}\left(s_{-i}, t\right)$ is upper hemi-continuous for each $i \in \mathcal{I}$. Let $h_{i}\left(S_{i}\right)$ be the image of the strategy set $S_{i}$ under $h_{i}(\cdot)$ and define the "reduced" best response correspondence $\tilde{R}_{i}\left(h_{T}(t)+\sum_{j \neq i} h_{j}\left(s_{j}\right)\right) \equiv R_{i}\left(s_{-i}, t\right)$ for each $i$. We can then define the following upper hemi-continuous (possibly empty-valued) correspondence:

$$
B_{\imath}(Q, t) \equiv\left\{\eta \in h_{i}\left(S_{i}\right): \eta \in h_{i} \circ R_{i}\left(h_{T}(t)+Q-\eta\right)\right\}
$$

for each player $i$. Let

$$
Z(Q, t) \equiv \sum_{j=1}^{I} B_{j}(Q, t)
$$

be the aggregate backward reply correspondence associated with the aggregate $Q=\sum_{j} h_{j}\left(s_{j}\right)$. Clearly, the "true" aggregate $g(s)=M\left(\sum_{j} h_{j}\left(s_{j}\right)\right)$ is monotonically increasing in the aggregate $Q=\sum_{j} h_{j}\left(s_{j}\right)$. Therefore, we may without loss of generality focus on $\sum_{j} h_{j}\left(s_{j}\right)$ instead of $g(s)$ in the following.

Let $q(Q, t) \in Z(Q, t)$ be the aggregate "Novshek-selection" shown as the thick segments in the figure below. 


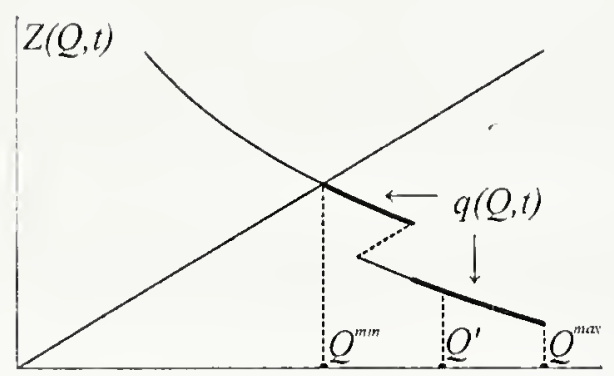

Figure 3: Constructing the aggregate "Novshek-selection"

A sketch of how this selection is constructed follows ${ }^{32}$ The first step is to pick $Q^{\text {max }}>0$, such that $q<Q^{\max }$ for all $q \in Z\left(Q^{\max }, t\right)$. To ensure that such a "point below the diagonal" exists, we can extend each of the correspondences $h_{i} \circ \tilde{R}_{i}$ along the lines of Kukushkin (1994) (see p. 24, 1.18-20). The details of this procedure need to be modified as follows. Let $D_{i}$ denote the subset of $\mathbb{R}$ upon which $h_{i} \circ \tilde{R}_{i}$ is defined, i.e., write $\gamma \in D_{i}$ if and only if $h_{i} \circ \tilde{R}_{i}(\gamma) \neq \emptyset$. Since $h_{i} \circ \tilde{R}_{i}$ is upper hemi-continuous, $D_{i}$ is closed. It is also a bounded set since $\tilde{R}_{i} \subseteq S_{i}$ and each $S_{i}$ is compact. Consequently, $D_{i}$ has a maximum, which we denoted by $d_{i}$. Then extend $h_{i} \circ \tilde{R}_{i}$ from $D_{i}$ to $D_{i} \cup\left(d_{i}, Q^{\max }\right]$ by taking $h_{i} \circ \tilde{R}_{i}(d) \equiv \perp_{i}$ all $d \in\left(d_{i}, Q^{\max }\right]$. Here $\perp_{i}$ can be any small enough element (for each player $i \in \mathcal{I}$ ) such that $\sum_{i} \perp_{i}<Q^{\max }, \perp_{i} \leq \min h_{i} \circ \tilde{R}_{i}\left(d_{i}\right)$, and $Q^{\max }-\perp_{i} \in\left(d_{i}, Q^{\max }\right]$. Defining the aggregate backward reply correspondence $Z$ as above, it is clear that $Z\left(Q^{\max }, t\right)=\sum_{i} \perp_{i}<Q^{\max }$.

Next let $D \subseteq\left(-\infty, Q^{m a x}\right]$ denote the subset of $\mathbb{R}$, where $Z(\cdot, t)$ is defined, i.e., those $Q \leq Q^{\text {max }}$ for which $B_{i}(Q, t) \neq \emptyset$, all $i$. The set $D$ is compact by the same argument as in the previous paragraph. Now, abusing notation slightly, let us denote a closed "interval" in $D$ by $\left[Q^{\prime}, Q^{\max }\right]$. That is, $\left[Q^{\prime}, Q^{\max }\right] \equiv D \cap\left\{Q: Q^{\prime} \leq Q \leq Q^{\max }\right\}$. Given such an interval $\left[Q^{\prime}, Q^{\max }\right]$, we say that a function $q:\left[Q^{\prime}, Q^{\max }\right] \times\{t\} \rightarrow \mathbb{R}$ is a maximal decreasing selection (from $Z$ ) if for all $Q \in\left[Q^{\prime}, Q^{\max }\right]$, the following are true: (i) $q(Q, t) \geq Z$ for all $Z \in Z(Q, t)\left(\subset 2^{\mathbb{R}}\right)$; (ii) $q(Q, t) \leq Q$; and (iii) the backward reply selections $b_{i}(Q, t) \in B_{i}(Q, t)$ associated with $q$ (i.e., backward by selections satisfying $q(Q, t)=\sum_{j} b_{j}(Q, t)$ all $Q$ ) are all decreasing in $Q$ on $\left[Q^{\prime}, Q^{\max }\right]\left(i . e ., Q^{\prime \prime}>Q^{\prime}\right.$ $\left.\Rightarrow b_{i}\left(Q^{\prime \prime}, t\right) \leq b_{i}\left(Q^{\prime}, t\right)\right)$.

Denote by $\Omega \subseteq 2^{\mathbb{R}}$ the set of all "intervals" $\left[Q^{\prime}, Q^{\max }\right]$ upon which a maximal decreasing selection exists. Notice that $\left\{Q^{\max }\right\} \in \Omega$ so $\Omega$ is not empty. $\Omega$ is ordered by inclusion since for any two elements $\omega^{\prime}, \omega^{\prime \prime}$ in $\Omega, \omega^{\prime \prime}=\left[Q^{\prime \prime}, Q^{\max }\right] \subseteq\left[Q^{\prime}, Q^{\max }\right]=\omega^{\prime} \Leftrightarrow Q^{\prime \prime} \leq Q^{\prime}$. A chain in $\Omega$ is a totally ordered subset (under inclusion). It follows directly from the upper hemi-continuity of the backward reply correspondences that any such chain with an upper bound has a supremum in $\Omega$ (i.e., $\Omega$ contains an "interval" that contains each "interval" in the chain). Zorn's Lemma therefore implies that $\Omega$ contains a maximal element, i.e., there exists an interval $\left[Q^{\text {min }}, Q^{\text {max }}\right] \in \Omega$ that is not (properly) contained in any other "interval" from $\Omega$.

The "Novshek-selection" is the maximal decreasing selection described above and defined on the interval $\left[Q^{\min }, Q^{\max }\right]$. See the figure.

The main step in proving the existence of equilibrium consists in showing that $Q^{\text {min }}$ is an equilibrium aggregate (it is easy to see that if $Q^{\min }$ is an equilibrium aggregate, then the associated backward replies form an equilibrium). Since we have $q\left(Q^{\min }, t\right) \leq Q^{\text {min }}$ by construction, this can be proved by showing that $q\left(Q^{\text {min }}, t\right)<Q^{\text {min }}$ cannot happen. This step is completed by showing that if $q\left(Q^{\min }, t\right)<Q^{\min }$ holds, then $q$ can be further extended "to the left" (and the

\footnotetext{
${ }^{32}$ The construction here is slightly different from the original one in Novshek (1955), but the basic intuition is the same. Aside from being somewhat briefer, the present way of constructing the "Novshek-selection" does not suffer from the "countability problem" in Novshek's proof pointed out by Kukuslikin (1994), since we use Zorn's Lemma for the selection.
} 
extension will satisfy (i)-(iii) above). This would violate the conclusion of Zorn's Lemma that $\left[Q^{\min }, Q^{\max }\right]$ is maximal, thus leading to a contradiction. The details of this step are identical to those in Novshek (1985) and are omitted.

Note that since $h_{T}$ is an increasing function, $q(Q, t)$ will be locally decreasing in $Q$ if and only if it is locally decreasing in $t$ (the effect of an increase in $t$ or $Q$ enter qualitatively in the same way in the definition of $B_{i}$, and so the effect of an increase on $q$ must be of the same sign). Figures 4-7 illustrate the situation for $t^{\prime}<t^{\prime \prime}$. The fact that the direction of the effect of a change in $Q$ and $t$ is the same accounts for the arrows drawn. In particular, any increasing segment will be shifted up and any decreasing segment will be shifted down because the shock hits the aggregate (this feature does not necessarily hold for other types of shocks).

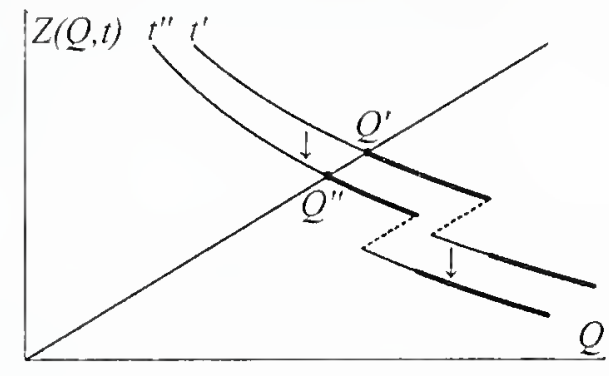

Figure 4: Case I

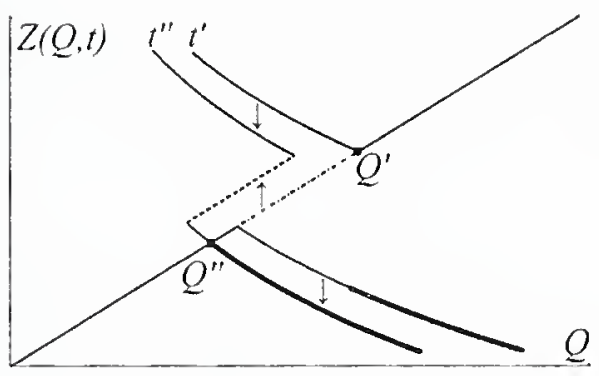

Figure 6: Case III

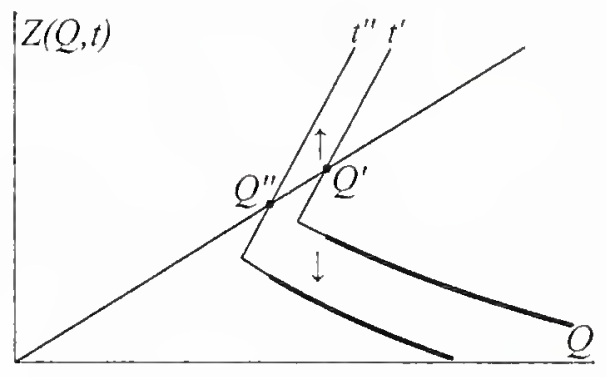

Figure 5: Case II

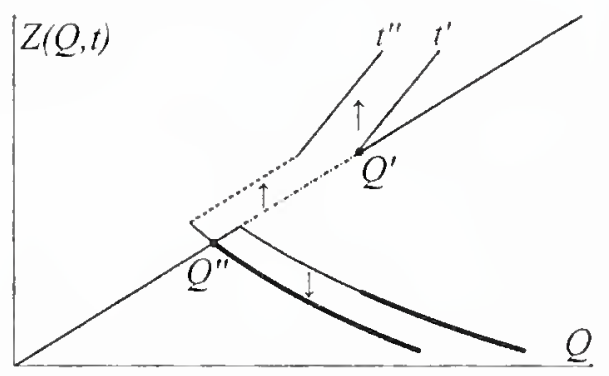

Figure 7: Case IV

There are four cases: Either the equilibrium aggregate varies continuously with $t$, and $q$ is decreasing (Case I) or increasing (Case II). Otherwise, the equilibrium aggregate "jumps" when $t$ is increased (Cases III and IV). [The figures depict the situations where the equilibrium aggregate jumps from $Q^{\prime}$ to $Q^{\prime \prime}$. This is associated with an increase in $t$. If instead $t$ decreases, case III reduces to Case I and Case IV reduces to Case II.]

Importantly, if the equilibrium aggregate jumps, it necessarily jumps down (and so is decreasing in $t$ ). The reason is that an increase in $t$ will always correspond to the graph of $q$ being shifted to "the left" (that is another way of saying that any increasing segment will be shifted up, and any decreasing segment shifted down). Hence no new equilibrium above the original largest one can appear, the jump has to be to a lower equilibrium. See the figures. We now consider Cases I and II in turn.

Case I: In this case we have $\underline{Q}<\bar{Q}$ such that $q(\underline{Q}, t)-\underline{Q}>0$ and $q(\bar{Q}, t)-\bar{Q}<0$, and such that the new equilibrium aggregate $Q^{\prime \prime}$ lies in the interval $[\underline{Q}, \bar{Q}]$. Since $q$ is decreasing in $t$, it trivially follows that $Q^{\prime \prime} \leq Q^{\prime}$. Note that this observation does not depend on continuity of $q$ in $Q$, but merely on the fact that a new equilibrium aggregate $Q^{\prime \prime}$ exists and lies in the neighborhood of $Q^{\prime}$ where $q$ is decreasing (in other words, it depends on the fact that the aggregate does not "jump"). 


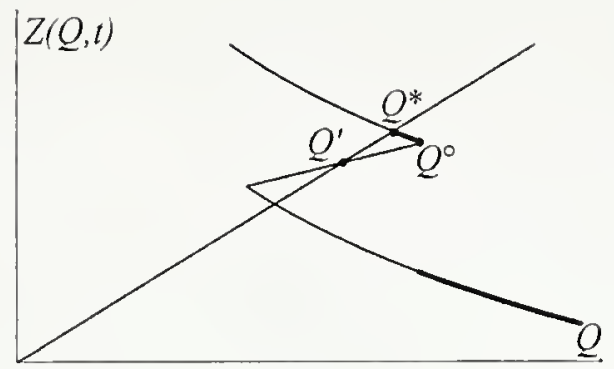

Figure 8: Slope below 1 is impossible: $Q^{\prime}$ being largest equilibrium aggregate violates that $q(Q, t)$ is decreasing in $Q$.

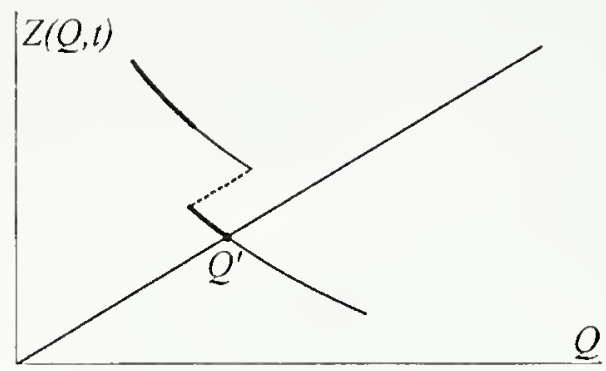

Figure 9: The "Novshek selection" leading to the smallest equilibrium aggregate.

Case II: As in Case I, we have $Q^{\prime \prime} \in[\underline{Q}, \bar{Q}]$. When $q$ is (locally) increasing, we must have $\underline{Q}<\bar{Q}$ such that $Q-q(Q, t)>0$ and $\bar{Q}-q(\bar{Q}, t)<0$. Intuitively, this means that the slope of $q$ is greater than 1 as illustrated in Figure 5. Indeed imagine that for the largest Nash equilibrium $Q^{\prime}$, there exists $Q^{\circ}>Q^{\prime}$ such that $Q^{\circ}-q\left(Q^{\circ}, t\right)<0$ (this means intuitively that the slope is below unity, see Figure 8). Then since $q\left(Q^{\circ}, t\right)>Q^{\circ}>Q^{\prime}$, no decreasing Novshek type selection could have reached $Q^{\prime}$ and there would consequently have to be a larger Nash equilibrium $Q^{*}$. This yields a contradiction.

We now argue that $Q^{\prime \prime} \leq Q^{\prime}$. As in the previous case, we prove this without explicit use of continuity (the proof is straightforward if continuity is used directly as seen in Figure 7). In particular, let us establish the stronger statement that $h_{T}(t)+Q$ is decreasing in $t$ (since $h_{T}(t$ ) is increasing in $t$, this implies that $Q$ must be decreasing in $t$ ). First define $C=h_{T}(t)+Q$, and consider the function $f(C, t)=C-h_{T}(t)-q\left(C-h_{T}(t), t\right)$. Let $\underline{C}=h_{T}(t)+Q$ and $\bar{C}=$ $h_{T}(t)+\bar{Q}$. By what was just shown, $f(\underline{C}, t)=\underline{Q}-q(\underline{Q}, t)>0$ and $f(\bar{C}, t)=\bar{Q}-\bar{q}(\bar{Q}, t)<0$. Since $B_{i}\left(C-h_{T}(t), t\right)$ is independent of $t$ ( $t$ cancels out in the definition of the backward reply correspondence), $q\left(C-h_{T}(t), t\right)$ must be constant in $t$, i.e., $q\left(C-h_{T}(t), t\right)=\tilde{q}(C)$ for some function $\tilde{q}$ which is increasing (since we are in Case II). So $f$ is decreasing in $t$ and $Q$, and we conclude that in equilibrium $f\left(C^{*}(t), t\right)=0, C^{*}(t)=h_{T}(t)+Q^{*}(t)$ is decreasing in $t$.

Remark 9 The fact that the term $h_{T}(t)+Q$ is decreasing in $t$ implies that, in this case, when there is entry of an additional player, the aggregate of all of the agents (including the entrant) decreases. To see this, compare with the proof of Theorem 5 and use that the aggregate of interest is $\sum_{j} h_{j}\left(s_{j}\right)+h_{I+1}\left(s_{I+1}\right)$ (in other words, take $h_{T}(t)=h_{I+1}\left(s_{I+1}\right)$ ).

Combining the observations made so far sliows that the largest equilibrium aggregate is decreasing in $t$ as claimed in the theorem. None of the previous conclusions depend on continuity of $q$ in $Q$, and it is straightforward to verify that the same conclusions hold regardless of whether $Q$ lies in a convex interval (strategy sets could be discrete, say). ${ }^{33}$ The statement for the smallest

\footnotetext{
${ }^{33}$ See Kukushkin (1994) for the details of how the backward reply selection is constructed in such non-convex cases.
} 
equilibrium aggregate can be shown by an analogous argument. In particular, instead of considering the selection $q(Q, t)$ one begins with $Q$ sufficiently low and studies the backward reply correspondence above the $45^{\circ}$ line, now choosing for every $Q$ the smallest best response (Figure 9). This completes the proof of Theorem 3.

\subsection{Proof of Theorem 5}

Let $\tilde{R}_{i}$ denote the "reduced" backward reply correspondence defined by $\tilde{R}_{i}\left(\sum_{j \neq i} h_{j}\left(s_{j}\right), t\right) \equiv$ $R_{i}\left(s_{-i}, t\right)$ for each $i$. To simplify notation, let us set $i=1$ (assume that the idiosyncratic shock hits the first player, in particular then $\tilde{R}_{i}$ is independent of $t=t_{1}$ for all $i \neq 1$ ). Any purestrategy Nash equilibrium will also be a fixed point of the set-valued equilibrium problem: $s_{1} \in$ $\tilde{R}_{1}\left(\sum_{j \neq 1} h_{j}\left(s_{j}\right), t_{1}\right)$ and $h_{i}\left(s_{i}\right) \in h_{i} \circ \tilde{R}_{i}\left(\sum_{j \neq i} h\left(s_{j}\right)\right)$ for $i=2, \ldots, I$. Consider the last $I-1$ inclusions, rewritten as

$$
h_{i}\left(s_{i}\right) \in h_{i} \circ \tilde{R}_{i}\left(\left(\sum_{j \neq i, 1} h_{j}\left(s_{j}\right)\right)+h_{1}\left(s_{1}\right)\right) \text { for } i=2, \ldots, I .
$$

For given $s_{1} \in S_{1}$, Theorem 3 implies that there exist a smallest and largest scalars $y_{*}\left(s_{1}\right)$ and $y^{*}\left(s_{1}\right)$ and solutions to the $I-1$ inclusions in (19) which $y_{*}\left(s_{1}\right)=\sum_{j \neq 1} h_{j}\left(s_{j, *}\right)$ and $y^{*}\left(s_{1}\right)=$ $\sum_{j \neq 1} h_{j}\left(s_{j}^{*}\right)$, respectively. In addition, $y_{*}, y^{*}: S_{1} \rightarrow \mathbb{R}$ are decreasing functions.

Now combining $y_{*}$ and $y^{*}$ that solve (19) in the sense described, with $s_{1} \in \tilde{R}_{1}\left(\sum_{j \neq 1} h_{j}\left(s_{j}\right), t_{1}\right)$ and replacing $s_{1}$ with $\bar{s}_{1}=-s_{1}$, we obtain a system with two inclusions:

$$
\bar{s}_{1} \in-\tilde{R}_{1}\left(y, t_{1}\right)
$$

and

$$
y \in\left\{y_{*}\left(-\bar{s}_{1}\right), y^{*}\left(-\bar{s}_{1}\right)\right\} .
$$

This system is ascending in $\left(\bar{s}_{1}, y\right)$ in the sense of Topkis (1998), hence its smallest and largest fixed points are decreasing in $t_{1}$ (since the system is descending in $t_{1}$ in the sense of Topkis). Therefore, the smallest and largest equilibrium strategies for player 1 are increasing in $t_{1}$, while the associated aggregate of the remaining players $y$ is decreasing in $t$. That the smallest and largest strategies for player 1 do in fact correspond to the smallest and largest strategies in the original game is straightforward to verify: Clearly, $y_{*}\left(s_{1}\right)$ and $y^{*}\left(s_{1}\right)$ are the smallest and largest aggregates of the remaining players (across all strategy profiles compatible with an equilibrium given $s_{1}$ ), and since $\tilde{R}_{1}$ is descending in $y$, the corresponding equilibrium values of $s_{1}$ are, respectively, the largest and the smallest.

Finally, it follows by construction that the corresponding aggregates of the remaining players must be the smallest and largest for the original game. This completes the proof of the theorem.

\subsection{Proof of Theorem 7}

We begin by noting that there is no loss of generality in using the aggregator $g(s) \equiv \sum_{2} h_{i}\left(s_{2}\right)$ in the following, and assuming that $\min _{s_{2} \in S_{2}} h_{\imath}\left(s_{\imath}\right)=0$ for all $i$. To see why, recall that the local solvability condition is independent of any strictly increasing transformation of the aggregator (Remark 5). If the original aggregator is $\tilde{g}(s)=H\left(\sum_{i} \tilde{h}_{i}\left(s_{i}\right)\right)$ we can therefore use the transformation $f(z)=H^{-1}(z)-\sum_{i} \min _{s_{i} \in S_{i}} \tilde{h}_{i}\left(s_{i}\right)$ to get the new aggregator $g(s) \equiv f(\tilde{g}(s))=\sum_{i} h_{i}\left(s_{i}\right)$, where $h_{i}\left(s_{i}\right) \equiv \tilde{h}_{i}\left(s_{i}\right)-\min _{s_{2} \in S_{2}} \tilde{h}_{i}\left(s_{i}\right)$. Clearly, $\min _{s_{2} \in S_{2}} h_{2}\left(s_{i}\right)=0$ for all $i$ so this new aggregator has all the desired properties. 
Let $R_{i}: S_{-i} \times T \rightarrow S_{i}$ be the best response correspondence of player $i$ and $\tilde{R}_{i}$ the reduced best response correspondence defined by $\tilde{R}_{i}\left(\sum_{j \neq i} h_{j}\left(s_{j}\right), t\right) \equiv R_{i}\left(s_{-i}, t\right)$. Define $\tilde{r}_{i}\left(\sum_{j \neq i} h_{j}\left(s_{j}\right), t\right) \equiv$ $h_{i} \circ \tilde{R}_{i}\left(\sum_{j \neq i} h_{j}\left(s_{j}\right), t\right)$, and write $\eta_{i} \in B_{i}(Q, t)$ whenever $\eta_{i} \in \tilde{r}_{i}\left(Q-\eta_{i}, t\right)$. It is clear that if $\eta_{i} \in B_{i}(Q, t)$ then there exists $s_{i} \in \tilde{R}_{i}\left(Q-h_{i}\left(s_{i}\right), t\right)$ with $\eta_{i}=h_{i}\left(s_{i}\right)$. So $Q$ is an equilibrium aggregate given $t \in T$ if and only if $Q \in Z(Q, t) \equiv \sum_{i} B_{i}(Q, t)$ (the correspondence $Z$ is the aggregate backward reply correspondence already studied in the proof of Theorem 3 ).

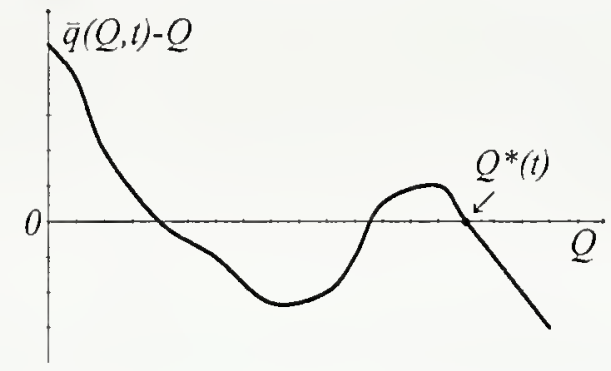

Figure 10: $Q^{*}(t)$ is the largest equilibrium aggregate given $t \in T$.

The main idea in the proof of Theorem 7 is to study the behavior of the smallest and a largest selections from $Z$, denoted below by $q(Q, t)$ and $\bar{q}(Q, t)$, respectively. Take the largest selection $\bar{q}(Q, t)$, and consider $\bar{q}(Q, t)-Q$ as a function of $Q$ (Figure 10). Any intersection with the first axis corresponds to an equilibrium aggregate since there $\bar{q}(Q, t)=Q$ and so $Q \in Z(Q, t)$. Since $\vec{q}$ is the largest selection from $Z$, the largest such intersection corresponds to the largest equilibrium aggregate $Q^{*}(t)$ as defined in (6). The desired result follows if we show that the equilibrium takes the form depicted in Figure 10 and in addition that $\bar{q}$ and $q$ are increasing in $t$. Indeed if this is the case, it is clear that any increase in $t$ will increase $Q^{*}(t)$. The same conclusion applies to $Q_{*}(t)$, the smallest intersection in an analogous figure, except with $\underline{q}(Q, t)-Q$ on the vertical axis rather than $\bar{q}(Q, t)-Q$. This is the conclusion of the theorem. The general features of $\bar{q}$ that ensured his conclusion are as follows. First, we must show that $\bar{q}$ is in fact well-defined on a compact interval in $Q$ (for all $t \in T$ ), below denoted $[\theta, \bar{Q}]$. This step is non-trivial and is the most difficult part of the proof. Second, we must show that $\bar{q}(Q, t)$ is continuous in $Q$. Third, we must show that $\vec{q}(Q, t)-Q$ "begins above the first axis and ends below it", i.e., we must show that $\bar{q}(\theta, t) \geq \theta$ and $\bar{q}(\bar{Q}, t) \leq \bar{Q}$. Finally, we must prove that $\bar{q}$ is increasing in $t$. In order to prove these claims, it turns out to be very convenient to introduce an index concept.

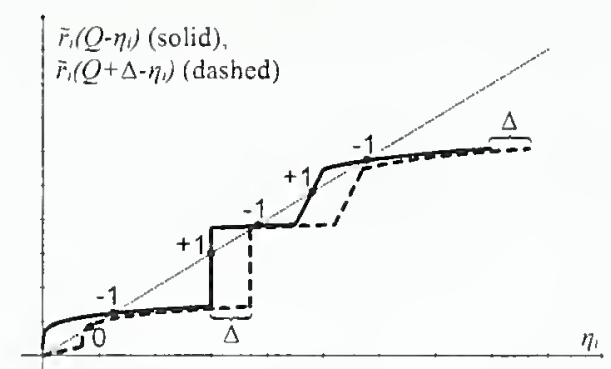

Figure 11: $\eta_{i}^{\prime} \in B_{\imath}(Q)$ if and only if the graph of $\tilde{r}_{i}\left(Q-\eta_{i}\right)$ intersects the diagonal at $\eta_{i}^{\prime}$.

Consider $\left(Q, \eta_{i}\right)$ with $\eta_{i} \in B_{i}(Q)$. We say that the index of such $\left(Q, \eta_{i}\right)$ is equal to $-1(+1)$ if and only if $\tilde{r}_{i}(Q-z)-z$ is strictly decreasing (strictly increasing) in $z$ on $\left(\eta_{i}-\epsilon\right) \cup\left(\eta_{i}+\epsilon\right), \epsilon>0$ (on the two one-sided open neighborhoods of $\eta_{\imath}$ ). If the index is not equal to either -1 or +1 , it is set equal to 0 . These indices are well-defined whether or not $\tilde{r}_{i}$ is single-valued at $Q-\eta_{i}$ since 
the local solvability condition implies that $\tilde{r}_{i}$ can be multivalued only at isolated points. ${ }^{34}$

Figure 11 illustrates the situation including the indices just defined. For $Q$ given, $\eta_{i} \in B_{i}(Q)$ if and only if the graph of $\tilde{r}_{i}(Q-\cdot)$ meets the diagonal. The dashed curve illustrates an important feature of $B_{i}$ : When the aggregate is changed from $Q$ to $Q+\Delta, \Delta>0$ say, this corresponds exactly to a parallel right transformation of $\tilde{r}_{i}(Q-\cdot)$ 's graph, i.e., every point on the curve in the figure is moved to derive by exactly an amount $\Delta>0$. This simple observation has several important consequences. (i) First, if any two points on the graph of $B_{\imath}$ lie on a $45^{\circ}$ line, then the whole interval between these two points must lie on the graph of $B_{i}$. In particular, if $\eta_{i} \in B_{i}(Q)$ and $\eta_{i}+\Delta \in B_{i}(Q+\Delta)$, then $\eta_{i}+\delta \in B_{i}(Q+\delta)$ for all $\delta \in[0, \Delta]$. (ii) Second, if $\eta_{i}^{\prime} \in B_{i}\left(Q^{\prime}\right)$ and the index of $\left(\eta_{i}^{\prime}, Q^{\prime}\right)$ is not zero, there exists a continuous function $f_{i}:\left(Q^{\prime}-\epsilon, Q^{\prime}+\epsilon\right) \rightarrow S_{i}$ such that $f_{i}(Q) \in B_{i}(Q)$ and $f_{i}\left(Q^{\prime}\right)=\eta_{i}^{\prime}$. In the following we shall refer to such a function as a continuous selection through $\left(\eta_{i}^{\prime}, Q^{\prime}\right)$. This extension procedure can be repeated as long as the index does not become equal to zero at any point or the boundary of $S_{i}$ is "hit" by the extension. Indeed, imagine that $f_{i}:\left(Q^{\prime}-\epsilon, Q^{\prime}+\epsilon\right) \rightarrow S_{i}$ is the continuous selection from before. By the upper hemi-continuity of $B_{i}$ (which follows straight from upper hemi-continuity of $\tilde{R}_{i}$ ) and the continuity of $f_{i}$ follows that $f_{i}$ can be extended to the boundary points, $f_{i}:\left[Q^{\prime}-\epsilon, Q^{\prime}+\epsilon\right] \rightarrow S_{i}$ in such a way that it is still true that $f_{i}(Q) \in B_{i}(Q)$ for all $Q$. As long as the index is non-zero on these boundary points, we can then take each of these as our starting point and start over in order to get a continuous selection $f_{i}:\left[Q^{\prime}-\epsilon^{\prime}, Q^{\prime}+\epsilon^{\prime}\right] \rightarrow S_{i}$ where $\epsilon^{\prime}>\epsilon$. An important thing to note about such a continuous selection that extends from an initial point $\left(\eta_{i}, Q\right)$ is that the index never changes along the selection: If the index of $\left(\eta_{i}, Q\right)$ is $-1(+1)$, the index of $\left.\left(f_{i}(Q), Q\right)\right)$ is $-1(+1)$ for all $Q$ at which the continuous selection is defined. (iii) Thirdly, because $\tilde{r}_{i}(Q) \geq 0$ for all $Q \geq 0$, the curve in Figure 11 always begins at $\eta_{i}=0$ at or above the diagonal. Hence it follows from the mean value theorem that if $\eta_{i}^{\prime} \in B_{i}\left(Q^{\prime}\right)$ for some $Q^{\prime}>0$, there will exist some $\eta_{i} \in B_{i}(Q)$ for all $Q>Q^{\prime}$. In other words: If $B_{i}\left(Q^{\prime}\right) \neq \emptyset$ for $Q^{\prime}>0$ then $B_{i}(Q) \neq \emptyset$ for all $Q>Q^{\prime}$ [When $Q$ is raised to $Q^{\prime}$ the point $\left(\eta_{i}^{\prime}, Q^{\prime}\right)$ is shifted "to the right" and so will lie below the $45^{\circ}$ line/diagonal. By continuity the curve must therefore intersect the diagonal in order to get from $\eta_{i}=0$ where it is above the diagonal to $\eta_{i}^{\prime}$ where it is below it]. (iv) Also from the fact that $\tilde{r}_{i}(Q) \geq 0$ for all $Q \geq 0$ follows that the index of the first intersection in the curve (corresponding to the smallest element in $\left.B_{i}(Q)\right)$ will be equal to either -1 or 0 whenever $\tilde{r}_{i}(Q)>0$. The case where $\tilde{r}_{i}(Q)=0$ is special and is considered in a footnote. ${ }^{35}(\mathrm{v})$ Finally, imagine that for some $Q^{\prime}, \eta_{i}^{\prime}=\max B_{i}\left(Q^{\prime}\right)$ (the largest elements in $B_{i}(Q) \subseteq \mathbb{R}_{+}$), and consider a continuous selection $f_{i}$ through $\left(\eta_{i}^{\prime}, Q^{\prime}\right)$. Again because the graph of $\tilde{r}_{i}$ is shifted to the right when $Q$ is raised, it is easy to verify from the figure that for all $Q>Q^{\prime}$ where $f_{i}$ is defined, $f_{\imath}(Q)=\max B_{i}(Q)$.

We are now ready to establish an important lemma.

Lemma 1 For each player $i \in \mathcal{I}$ there exists a finite number of continuous functions $b_{i, 1}, \ldots, b_{i, M_{2}}$ each defined on $\left[\theta_{i}, \bar{Q}\right]$ where $\theta_{i} \equiv \min _{s_{i} \in S_{2}} \tilde{r}_{i}(0)$ and $\bar{Q} \equiv \max \left\{\sum_{i} h_{i}\left(s_{i}\right) \in \mathbb{R}_{+}: s \in S\right\}$ and taking values in $\mathbb{R}_{+}$, such that for every $Q \in[0, \bar{Q}], B_{\imath}(Q)=\cup_{m=1}^{M_{2}} b_{i, m}(Q)$ (in particular $B_{\imath}(Q)=\emptyset$ for $\left.Q<\theta_{i}\right)$. In addition, for $m \neq m^{\prime} \in\left\{1, \ldots, M_{\imath}\right\}$ it cannot happen that $b_{i, m}(Q)=b_{\imath, m^{\prime}}(Q)$ for any $Q$ with $b_{i, m}(Q)>\min _{s_{2} \in S_{i}} h_{i}\left(s_{i}\right)$.

Proof. By construction $\theta_{i} \in B_{i}\left(\theta_{i}\right)$. We are first going to show that $B_{i}(Q)=\emptyset$ for all $Q<\theta_{i}$. Clearly, for $Q<\theta_{i}$ there cannot exist $\eta_{i} \in B_{i}(Q)$ with $\eta_{i}>Q$ (indeed for such $\eta_{i}$ and $Q$ we would

\footnotetext{
${ }^{34}$ Precisely, if $\tilde{r}_{i}(Z)$ is not single-valued at $Z$, it must be single-valued on a sufficiently small neighborhood of $Z$. This is a consequence of local solvability because this condition implies that for $Q$ fixed, any solution to $\eta_{i} \in \vec{r}_{2}\left(Q-\eta_{i}\right)$ must be locally unique. Indeed, $\eta_{i} \in \tilde{r}_{i}\left(Q-\eta_{i}\right) \Leftrightarrow\left[\eta_{i}=h_{i}\left(s_{i}\right) \& \Psi_{i}\left(s_{i}, Q\right)=0\right]$ and $s_{i}$ is locally uniquely determined as a function of $Q$ when $\left|D_{s_{2}} \Psi_{\imath}\right| \neq 0$ (see, e.g., Milnor (1965), p.8).

${ }^{35}$ When $\tilde{r}_{2}(Q)=0$ it is possible that the curve intersects the diagonal at $\left(\eta_{2}, \tilde{r}_{2}\left(Q-\eta_{2}\right)\right)=(0,0)$ and "stays above" the diagonal for all $\eta_{2}>0$ in a neighborhood of 0 . If we extend our index definition above to boundary points in the natural way, this intersection would have index +1 (the natural way to extend to a boundary point is to require that the original index definition holds on a suitably restricted one-sided neighborhood).
} 
have to have $s_{i} \in \tilde{R}_{i}\left(Q-\eta_{i}\right)$ where $Q-\eta_{i}<0$. But $\tilde{R}_{i}$ is only defined on $\left.\sum_{j \neq i} h_{j}\left(S_{j}\right) \subseteq \mathbb{R}_{+}\right)$. If $Q<\theta_{i}$ and $\eta_{i} \in B_{i}(Q)$, we must therefore have $\eta_{i}<Q$ (we cannot have equality because then $\theta_{i}$ would not be the minimum of $\left.\tilde{r}_{i}(0)\right)$. So assume, ad absurdum that $\eta_{i}<Q$. Let $s_{i}$ be such that $s_{i} \in \tilde{R}_{i}\left(Q-\eta_{i}\right)$ (in particular then $\eta_{i}=h_{i}\left(s_{i}\right)$ ). Unless $s_{i}$ is on the boundary of $S_{i}$, we have $\Psi_{i}\left(s_{i}, Q\right)=0$ since first-order conditions are necessary for an interior solution. By the local solvability condition therefore $\left|D_{s_{i}} \Psi_{i}\left(s_{i}, Q\right)\right| \neq 0$ which implies that there exists a "downwards" continuous selection through $\left(\eta_{i}, Q\right), f_{i}:(Q-\epsilon, Q] \rightarrow S_{i}$. At $\left(f_{i}(Q-\epsilon), Q-\epsilon\right)$ we again have (unless $f_{i}(Q)=h_{i}\left(s_{i}\right)$ where $s_{i}$ is a boundary point and $\Psi_{i}\left(s_{i}, Q\right)=0$ does not hold) that the local solvability condition applies and so we can extend further downwards. This process continues until we reach a point $Q^{\prime}<Q$ such that any $s_{i} \in S_{i}$ with $f_{i}\left(Q^{\prime}\right)=h_{i}\left(s_{i}\right)$ is a boundary point of $S_{i}$. If the index of $\left(\theta_{i}, \theta_{i}\right)$ is equal to +1 , we can begin the above selection procedure from $\left(\theta_{i}, \theta_{i}\right)$ and will, as mentioned, eventually reach a boundary point in the above sense. But when this selection first "hits" the boundary $\Psi_{i}\left(s_{i}, Q\right)=0$ still holds ( $\Psi_{i}$ is continuous), and so from the local solvability condition follows that the index is (still) +1 . Because of (i) above, this selection is the maximal selection: There will be no $\tilde{\eta}_{i} \in B_{i}(Q)$ with $\tilde{\eta}_{i}>f_{i}(Q)$ for any $Q$ where $f_{i}$ is defined. $^{36}$ If the index of $\left(\theta_{i}, \theta_{i}\right)$ is either 0 or -1 , we can either still construct a continuous downwards selection through $\left(\theta_{i}, \theta_{i}\right)$ as the one above (precisely this happens in the index is 0 and the graph of $\tilde{r}_{i}\left(\theta_{i}-\cdot\right)$ intersects the diagonal from below), or else we can begin at $\left(\eta_{i}, Q\right)$ (where as before $Q<\eta_{i}$ ) and construct a similar maximal extension. Precisely this happens either if the index of $\left(\theta_{i}, \theta_{i}\right)$ is -1 or if the index is 0 and the graph of $\tilde{r}_{i}\left(\theta_{i}-\cdot\right)$ intersects the diagonal from above. In either case the index of the downward selection through $\left(\eta_{i}, Q\right)$ must be +1 so this leads to a situation that is entirely symmetric with the previous one.

In sum, at $Q^{\prime}$ there is exactly one solution to the equation $\Psi_{i}\left(s_{i}, Q^{\prime}\right)=0$ and here $h_{i}\left(s_{i}\right)=$ $f_{i}\left(Q^{\prime}\right)$. And the index of $\left(f_{i}\left(Q^{\prime}\right), Q^{\prime}\right)$ is equal to +1 . Since $\tilde{r}_{i}(Q) \geq 0$ for any $Q \geq 0$, this implies that a selection from $B_{i}$, different from $f_{i}$, exists on $\left[Q^{\prime}, Q^{\prime}+\epsilon\right], \epsilon>0$ (recall from (iv) above that the index of the smallest selection from $B_{i}$ is -1 unless possibly if $\tilde{r}_{i}(0)=0$. But $\tilde{r}_{i}(0)=0$ cannot happen unless $\theta_{i}=0$ in which case the claim that $B_{i}(Q)=\emptyset$ for $Q<\theta_{i}$ is vacuous). This selection is of course locally non-decreasing (possibly constant). Importantly, it necessarily has index equal to -1 . But then two selections with different indices "meet" at $Q$ '. This can never happen if $\Psi_{i}\left(s_{i}, Q^{\prime}\right)=0$ and $z^{\prime}=h_{i}\left(s_{i}\right)$ hence we have arrived at a contradiction if payoff functions are assumed to satisfy the first-order conditions at any best response for the players. If not, we have a contradiction with regularity which specifically says that there does not exist an aggregate such as $Q^{\prime}$ above where all backward replies' indices are positive.

We conclude that $B_{i}(Q)=\emptyset$ for $Q<\theta_{i}$ which is what we set out to do. Along the way, we also ended up concluding that the index of $\left(\theta_{i}, \theta_{i}\right)$ cannot be +1 , nor can it be 0 if the graph of $\tilde{r}_{i}\left(\theta_{i}-\cdot\right)$ intersects the diagonal from below. In either case, this implies that we can find a continuous upwards selection through $\left(\theta_{i}, \theta_{i}\right)$. This selection has index -1 whenever $f_{i}(Q)$ is such that $f_{i}(Q)=h_{i}\left(s_{i}\right)$ where $s_{i}$ is in the interior of $S_{i}$ (in fact, it is sufficient that the first order conditions hold, so this is always true in the multidimensional case and also in the one-dimensional case unless $f_{i}(Q)$ is horizontal, in which case the statements below become trivial). Furthermore, since this selection is maximal at $\theta_{i}$ it remains the maximal selection from $B_{i}$ for all $Q>\theta_{i}$ (this follows from (v) above). Finally, the selection will actually exist on all of $\left[\theta_{i}, \bar{Q}\right]$ because the index is equal to -1 throughout (see also (iii) above). In term of the lemma's statement, we have therefore found our first function $b_{i, 1}:\left[\theta_{i}, \bar{Q}\right] \rightarrow \mathbb{R}_{+}$, namely the maximal selection from $B_{i}$ that intersects at $\left(\theta_{i}, \theta_{i}\right)$.

Even under the local solvability condition, we cannot conclude that this is the only selection

\footnotetext{
${ }^{36}$ Imagine such a larger element in $B_{2}$ did exist, $\tilde{\eta}_{2} \in B_{2}(Q)$. This would imply that the whole $45^{\circ}$ segment between $\left(\tilde{\eta}_{i}, Q\right)$ and the intersection of the graph of $f_{2}$ with the $45^{\circ}$ line through $\left(\tilde{\eta}_{2}, Q\right)$ would lie in $B_{2}$ 's graph. But this is a contradiction because at the point where the $45^{\circ}$ segment intersects with the graph of $f_{2}$, local solvability would be violated (local solvability implies, in particular, local uniqueness of selections, see footnote 34 for a similar argument).
} 
from $B_{i}$. Local solvability does, however, imply that the number of selections must be finite (or else they would not be locally isolated which is a fact we have used several times above). In addition, local solvability rules out that any selection has index 0 when $\Psi_{i}\left(s_{i}, Q\right)=0$ for $s_{i}$ with $f_{i}(Q)=h_{i}\left(s_{i}\right)$. Hence the indices must be alternating in sign when ordered in the natural way (the greatest selection above has index -1 , the next selection below it has index +1 , etc., until the least selection which always has index -1 ). Also from local solvability follows that two selection cannot intersect each other (except possibly at the "lower boundary" and not at all when $N>1$ ). If they did intersect, $i . e$. , if for any two selections $m \neq m^{\prime} \in\left\{1, \ldots, M_{i}\right\}$ we had $b_{i, m}(Q)=b_{i, m^{\prime}}(Q)$ for any $Q$ with $b_{i, m}(Q)>\min _{\mathcal{s}_{i} \in S_{i}} h_{i}\left(s_{i}\right)$, these selection would either have to be equal everywhere (and so there is only one!), or else they would at some point deviate from each other and at that point local uniqueness of selections (explained several times above) would be violated.

What remains to be shown now is only that all selections are defined on $\left[\theta_{i}, \bar{Q}\right]$. This has already been shown to be the case for the greatest selection $b_{i, 1}$. That it is also true for the other selections follows from a similar argument recalling that the indices must be alternating and that the index of the least selection is -1 . The details are omitted.

Let $\mathcal{M}_{i} \equiv\left\{1, \ldots, M_{i}\right\}$ where $M_{i}$ is the number of functions of the previous lemma. Now making the dependence on $t$ explicit, we define

$$
\bar{q}(Q, t) \equiv \max _{\left(m_{1}, \ldots, m_{I}\right) \in \mathcal{M}_{1} \times \ldots \times \mathcal{M}_{I}} \sum_{i} b_{i, m_{\imath}}(Q, t) .
$$

Clearly $\bar{q}(Q, t)$ is only defined on $[\theta, \bar{Q}]$ where $\theta \equiv \max _{i} \theta_{i}$. It is also clear that $\bar{q}$ takes values in the interval $[0, \bar{Q}]$. From now on we focus on $\bar{q}$, which will prove the statement of Theorem 7 for the largest equilibrium aggregate. For the smallest aggregate one considers instead $q(Q, t)$ defined as above replacing max with min and proceeds as below with the obvious modifications.

The following lemma will essentially finish the proof of the theorem by establishing all of the claims mentioned in our outline of the proof above.

Lemma 2 The function $\bar{q}:[\theta, \bar{Q}] \times T \rightarrow[0, \bar{Q}]$ is continuous in $Q$ on $[\theta, \bar{Q}]$, increasing in $t$ on $T$, and satisfies $\bar{q}(\theta) \geq \theta$ (of course we also have $\bar{q}(\bar{Q}, t) \leq \bar{Q}$ ).

Proof. Continuity of $\bar{q}$ in $Q$ follows directly from the definition of $\bar{q}$ and lemma 1 . Let $m^{\prime}(i)$ denote the index of the selection that solves (11.4) for player $i \in \mathcal{I}$. In the notation of the proof of Lemma $1, m^{\prime}(i)$ must in fact be equal to 1 for every player (the greatest selection). For this greatest selection, it was shown in the proof of the Lemma that $b_{i, 1}\left(\theta_{i}, t\right)=\theta_{i}$. It follows that $\bar{q}(\theta, t)=\sum_{i} b_{i, 1}(\theta, t) \geq b_{i^{\prime}, 1}(\theta, t)=\theta$ where $i^{\prime}$ is any player for which $\theta_{i}=\theta$. This proves the last claim of the Lemma. Finally, we show that $\bar{q}$ is increasing in $t$. For any $Q, \eta_{i}=b_{i, m^{\prime}(i)}(Q, t)$ if and only if $\eta_{i}$ is the largest solution to the fixed point problem: $\xi_{i} \in \tilde{r}_{i}\left(Q-\xi_{i}, t\right)$. By assumption, $t$ is a positive shock in the sense that the smallest and largest selections of $\tilde{r}_{i}\left(Q-\xi_{i}, t\right)=h_{i} \circ \tilde{R}_{2}\left(Q-\xi_{i}, t\right)$ are increasing in $t$ (for all fixed $Q$ and $\xi_{i}$ ). Moreover, the smallest (respectively, the largest) selection from an upper hemi-continuous correspondence with range $\mathbb{R}$ is lower semi-continuous (respectively, upper semi-continuous). ${ }^{37}$ In particular, the least selection is "lower semi-continuous from above" and the greatest selection is "upper semi-continuous from below". Hence any upper hemi-continuous and convex valued correspondence with values in $\mathbb{R}$ satisfies the conditions of

\footnotetext{
${ }^{37}$ Let $F: X \rightarrow 2^{\mathbb{R}}$ be such a correspondence, and $f_{*}$ and $f^{*}$ the smallest and largest selections, i.e., $f^{*}(x) \equiv$ $\max _{z \in F(x)} z$ and $f_{*}(x) \equiv-\left[\max _{z \in-F(x)} z\right]$. Since the value function of a maximization problem is upper semicontinuous when the objective function is continuous and the constraint correspondence is upper hemi-continuous, it follows that $f^{*}$ is upper semi-continuous and moreover $\tilde{f}_{\bullet}(x)=\max _{z \in-F(x)} z$ is upper semi-continuous, thus implying that $f_{*}=-\tilde{f}$. is lower semi-continuous.
} 
Corollary 2 in Milgrom and Roberts (1994). We therefore conclude that $b_{i, m^{\prime}(i)}(Q, t)$ is increasing in $t$ (and likewise that the smallest fixed point of $\xi_{i} \in \tilde{r}_{i}\left(Q-\xi_{i}, t\right)$ is increasing in $t$ which one uses to prove the theorem's statement for the smallest aggregate). Clearly then, $\bar{q}(Q, t)$ is also increasing in $t$.

As was mentioned above, when the continuous function $\bar{q}(Q, t)$ (where $t$ is fixed) is shifted everywhere up because $t$ is increased, the largest solution of the equation $Q=\bar{q}(Q, t)$ must increase (and for the smallest aggregate the same conclusion applies to the smallest solution of $q(Q, t)=Q)$. This completes the proof of the theorem.

\subsection{Proof of Theorem 8}

The statement is proved only for the smallest equilibrium aggregate (the proof for the largest aggregate is similar). Let $b_{m^{\prime}}$ be the least backward reply map as described in the proof of Theorem 7. Let us define $t(I+1)=s_{I+1}^{*} \in \mathbb{R}_{+}^{N}$ (i.e., as equal to the entrant's equilibrium strategy) and $t(I)=0$. Then $Q_{*}(I)$ and $Q_{*}(I+1)$ are the least solutions to, $Q(I)=g(b(Q(I))$, $t(I))$ and $Q(I+1)=g(b(Q(I+1)), t(I+1))$, respectively. Since $g$ is increasing in $t$ and $t(I) \leq t(I+1)$, this is a positive shock to the aggregate backward reply map. That $Q_{*}(I) \leq Q_{*}(I+1)$ must hold then follows by the same arguments as in the proof of Theorem 7. Clearly, $Q_{*}(I)=Q_{*}(I+1)$ cannot hold unless $t(I)=t(I+1)$ since $g$ is strictly increasing.

\subsection{Proof of Proposition 3}

We begin by verifying the local solvability condition in the contest game of Proposition 3. Direct calculations yield

$$
\Psi_{i}\left(s_{i}, Q\right)=V_{i} \cdot\left[\frac{h_{i}^{\prime}\left(s_{i}\right)}{R+Q}-\frac{H^{\prime}\left(H^{-1}(Q)\right) h_{i}^{\prime}\left(s_{i}\right) h_{i}\left(s_{i}\right)}{(R+Q)^{2}}\right]-c_{i}^{\prime}\left(s_{i}\right),
$$

and

$$
\begin{aligned}
D_{s_{i}} \Psi_{i}= & \frac{V_{i} h_{i}^{\prime \prime}\left(s_{i}\right)}{h_{i}^{\prime}\left(s_{i}\right)} \cdot\left[\frac{h_{i}^{\prime}\left(s_{i}\right)}{R+Q}-\frac{H^{\prime}\left(H^{-1}(Q)\right) h_{i}^{\prime}\left(s_{i}\right) h_{i}\left(s_{i}\right)}{(R+Q)^{2}}\right] \\
& -c_{i}^{\prime \prime}\left(s_{i}\right)-V_{i} \frac{H^{\prime}\left(H^{-1}(Q)\right)\left(h_{i}^{\prime}\left(s_{i}\right)\right)^{2}}{(R+Q)^{2}} .
\end{aligned}
$$

Therefore, when $\Psi_{i}\left(s_{i}, Q\right)=0$, we have

$$
D_{s_{i}} \Psi_{i}=\frac{h_{i}^{\prime \prime}\left(s_{i}\right)}{h_{i}^{\prime}\left(s_{i}\right)} c_{i}^{\prime}\left(s_{i}\right)-c_{i}^{\prime \prime}\left(s_{i}\right)-V_{i} \cdot \frac{H^{\prime}\left(H^{-1}(Q)\right)\left(h_{i}^{\prime}\left(s_{i}\right)\right)^{2}}{(R+Q)^{2}} .
$$

Dividing both sides by $c_{i}^{\prime}\left(s_{i}\right)>0$, we conclude that $D_{s_{2}} \Psi_{i}<0$, and thus the local solvability condition is satisfied and each $\Psi_{i}$ is regular.

It is also straightforwald to verify that $\tilde{c}_{\imath}=c_{i} \circ h_{i}^{-1}$ is a convex function under our hypotheses.

Next, consider the payoff function of player $i$ after the change of coordinates $s_{i} \mapsto z_{i}=h_{i}\left(s_{i}\right)$ (for $i \in \mathcal{I}): \tilde{\pi}_{i}(z)=V_{i} z_{i}\left[R+H\left(\sum_{j=1}^{I} z_{j}\right)\right]^{-1}-\tilde{c}_{i}\left(z_{i}\right)$. If $D_{z_{i}} \tilde{\pi}_{i}(z)=0$ is sufficient to ensure that $z_{i}$ is a global maximum, then the original first-order condition are also sufficient for a global maximum. In particular, $D_{z_{2}} \tilde{\pi}_{i}(z)=0$ implies that $D_{z_{2}^{2}}^{2} \tilde{\pi}_{\imath}(z)<0$. Hence any interior extremum is a strict maximum, from which follows that there is at most one extremum, necessarily a global 
maximum (it is possible that this maximum is on the boundary, though in this case it continues to be unique; see also Remark 3).

Now, parts 1 and 2 the proposition follow directly from Theorems 7 and 8 . Part 3 follows from Theorem 9 by noting that the condition for $s_{i}^{*}(t)$ to be locally increasing in a positive shock $t$ is

$$
-\left[D_{s_{i}} \Psi_{i}\left(s_{i}^{*}, g\left(s^{*}\right), t\right)\right]^{-1} D_{Q} \Psi_{i}\left(s_{i}^{*}, g\left(s^{*}\right), t\right) \geq 0 .
$$

Since, as shown above, $D_{s_{i}} \Psi_{i}\left(s_{i}^{*}, g\left(s^{*}\right), t\right)<0,(20)$ holds if and only if $D_{Q} \Psi_{i}\left(s_{i}^{*}, Q^{*}, t\right) \geq 0$ where $Q^{*}=g\left(s^{*}\right)$. For the same reason, the condition for $s_{i}^{*}(t)$ to be decreasing in $t$ when $t$ does not directly affect player $i$ (the second statement of part 3 ), is satisfied is and only if $D_{Q} \Psi_{i}\left(s_{i}^{*}, Q^{*}\right) \leq 0$.

Since,

$$
\begin{aligned}
& D_{Q} \Psi_{i}\left(s_{i}^{*}, Q^{*}, t\right)=V_{i} \times \\
& \quad\left[-\frac{h_{i}^{\prime}\left(s_{i}^{*}\right)}{\left(R+Q^{*}\right)^{2}}+\frac{2 H^{\prime}\left(H^{-1}\left(Q^{*}\right)\right) h_{i}^{\prime}\left(s_{i}^{*}\right) h_{i}\left(s_{i}^{*}\right)}{\left(R+Q^{*}\right)^{3}}-\frac{H^{\prime \prime}\left(H^{-1}\left(Q^{*}\right)\right)\left(H^{-1}\right)^{\prime}\left(Q^{*}\right) h_{i}^{\prime}\left(s_{i}^{*}\right) h_{i}\left(s_{i}^{*}\right)}{\left(R+Q^{*}\right)^{2}}\right],
\end{aligned}
$$

(20) will hold if and only if

$$
h_{i}\left(s_{i}^{*}\right) \geq \eta\left(Q^{*}\right)
$$

where

$$
\eta\left(Q^{*}\right) \equiv\left[\frac{2 H^{\prime}\left(H^{-1}\left(Q^{*}\right)\right)}{\left(R+Q^{*}\right)}-\frac{H^{\prime \prime}\left(H^{-1}\left(Q^{*}\right)\right)}{H^{\prime}\left(H^{-1}\left(Q^{*}\right)\right)}\right]^{-1} .
$$

This shows that player $i$ will increase its effort if it is "dominant" as defined in the proposition. If instead $h_{i}\left(s_{i}^{*}\right) \leq \eta\left(Q^{*}\right)$, i.e., if the player is not "dominant", $D_{Q} \Psi_{i}\left(s_{i}^{*}, Q^{*}\right) \leq 0$ and by Theorem 9 follows that if the player is not affected by the shock, she will decrease her effort in equilibrium.

\subsection{Proof of Theorem 11}

We begin with a technical lemma:

Lemma 3 Suppose Assumption 1 holds. Then:

(i) $\left[D_{s_{i}} \Psi_{i}\left(s_{i}, \sum_{j=1}^{I} s_{j}, t\right)\right]^{-1}$ exists and all of its elements are non-positive; and

(ii) $D_{s_{2} s_{2}}^{2} \Pi_{i}\left(s_{i}, \sum_{j=1}^{I} s_{j}, t\right)$ is negative definite.

Proof. For a matrix A with non-negative off-diagonal entries the following four statements are equivalent (see Berman and Plemmons (1994), pages 135-136): (1) all eigenvalues of A have negative real parts; (2) all real eigenvalues of $\mathbf{A}$ are negative; (3) there exists a vector $x \in \mathbb{R}_{++}^{N}$ such that $\mathrm{A} x \in \mathbb{R}_{--}^{N} ;$ (4) $\mathrm{A}^{-1}$ exists and all of its elements are non-positive.

It is clear from (16) that if $D_{s_{i} s_{i}}^{2} \Pi_{i}$ has non-negative off-diagonal entries and $D_{Q} \Psi_{i}$ is nonpositive, then $D_{s_{i}} \Psi_{i}$ must have non-negative off-diagonal entries. By assumption, all real eigenvalues

$D_{s_{2}} \Psi_{i}\left(s_{i}, \sum_{j=1}^{I} s_{j}, t\right)$ are negative, hence (4) holds verifying the first claim of the lemma. For the second claim, we use that (3) holds for $D_{s_{2}} \Psi_{i}$, and let $x \in \mathbb{R}_{++}^{N}$ be such that $D_{s_{1}} \Psi_{i} \cdot x \in$ $\mathbb{R}_{--}^{N}$. Clearly $D_{Q} \Psi_{i} \cdot x \in \mathbb{R}_{-}^{N}$ because $D_{Q} \Psi_{i}$ is non-positive. Hence from (16) follows that $D_{s_{2} s_{i}}^{2} \Pi_{2}\left(s_{i}, \sum_{j=1}^{I} s_{j}, t\right) x \in \mathbb{R}_{--}^{N}$. But then (since $D_{s_{i} s_{2}}^{2} \Pi_{i}$ has non-negative off-diagonal elements) 
all of its eigenvalues have negative real parts, and being symmetric it is therefore negative definite.

Next note that since $D_{Q} b=-\left[D_{s_{i}} \Psi_{i}\right]^{-1} D_{Q} \Psi_{i}$, part (i) of Lemma 3 implies that the backward reply function $b$ is decreasing in $Q$ (since $D_{Q} \Psi_{i}$ is a non-positive matrix in view of the fact that the payoff function exhibits decreasing differences).

Finally, to establish the main result, differentiate $Q=b(Q+t)$ to obtain:

$$
d Q=D_{Q} b(Q+t) d Q+D_{Q} b(Q+t) d t
$$

Since $D_{Q} b(Q+t)$ is non-singular, this is equivalent to

$$
\left[\left[D_{Q} b(Q+t)\right]^{-1}-\mathbf{I}\right] d Q=d t .
$$

The sufficiency part of the theorem will follow if we can show that $d t \geq 0 \Rightarrow d Q \leq 0$. By the previous equation, this is equivalent to: $\left[\left[D_{Q} b(Q+t)\right]^{-1}-\mathbf{I}\right] d Q \geq 0 \Rightarrow d Q \leq 0$. An alternative (but again equivalent) way of writing this is,

$$
\left[\mathrm{I}-\left[D_{Q} b(Q+t)\right]^{-1}\right] d Q \geq 0 \Rightarrow d Q \geq 0 .
$$

The statement in (21) is very well known in matrix algebra: a matrix $\mathbf{A}$ such that $A x \geq 0 \Rightarrow x \geq 0$ is called a monotone matrix (Berman and Plemmons (1994)). A well known result from matrix algebra tells us that a matrix is monotone if and only if it is a non-singular $M$-matrix (Berman and Plemmons (1994), page 137). Since $\left[\mathbf{I}-\left[D_{Q} b(Q+t)\right]^{-1}\right]$ is non-singular by assumption, it is a non-singular $M$-matrix when it is an $M$-matrix (as assuned in the theorem). Hence, it will be monotone and so any small increase in $t$ (in one or more coordinates) will lead to a decrease in each of Q's coordinates.

As for the theorem's necessity statement, assume that $\left[\mathrm{I}-\left[D_{Q} b(Q+t)\right]^{-1}\right]$ is not an $M$-matrix. By the result just used, this is the same as saying that $\left[\mathbf{I}-\left[D_{Q} b(Q+t)\right]^{-1}\right]$ is not monotone, which implies that $d Q \leq \leq 0$ and $\left[\mathbf{I}-\left[D_{Q} b(Q+t)\right]^{-1}\right] d Q \leq 0$ for at least one vector $d Q \in \mathbb{R}^{N}$. We cannot have $\left[\mathbf{I}-\left[D_{Q} b(Q+t)\right]^{-1}\right] d Q=0$ since $\mathbf{I}-\left[D_{Q} b(Q+t)\right]^{-1}$ is non-singular; hence $\left[\mathrm{I}-\left[D_{Q} b(Q+t)\right]^{-1}\right] d Q<0$ for some such vector $d Q \leq 0$. Now we simply pick $t^{\prime \prime}-t^{\prime}=d t=$ $-\left[\mathbf{I}-\left[D_{Q} b(Q+t)\right]^{-1}\right] d Q>0$ and the associated change in the aggregate $d Q$ will then be increasing in at least one coordinate/component, which is the statement of the theorem.

\subsection{Proof of Theorem 14}

We begin with Theorem 7. To simplify the notation in the proof, let us focus on the case with a linear aggregator, $g(s)=\sum_{j=1}^{I} s_{j}$ (the general case is proved by the exact same argument, the only difference is that it becomes very notation-heavy). Then, for each $i, G_{i}(Q, y)=Q-y$. Define $M_{i}(y, t) \equiv \arg \max _{Q \geq y} \Pi_{i}(Q-y, Q)$. Clearly, $M_{i}(y, t)-\{y\}=\tilde{R}_{i}(y, t)$, where $\tilde{R}_{i}$ is the "reduced" best response correspondence (i.e., best response as a function of the sum of the opponents' strategies). Hence, we can write $M_{i}\left(Q-s_{i}, t\right)-\{Q\}=\tilde{R}_{i}\left(Q-s_{i}, t\right)-\left\{s_{i}\right\}$. Given singlecrossing, $M_{i}(y, t)$ is ascending in $y$ (e.g., Milgrom and Shannon (1994), Theorem 4). Therefore, $\tilde{R}_{i}\left(Q-s_{i}, t\right)-\left\{s_{i}\right\}$ must be descending in $s_{i}$. Moreover, $\tilde{R}_{i}\left(Q-s_{i}, t\right)-\left\{s_{i}\right\}$ is convex-valued. Let $B_{i}(Q, t)=\left\{s_{i} \in S_{i}: s_{i} \in \tilde{R}_{i}\left(Q-s_{i}, t\right)\right\} . B_{i}(Q, t) \neq \emptyset$ since: $z \in \tilde{R}_{i}\left(Q-\perp_{i}, t\right) \Rightarrow z-L_{i} \geq 0$ (where $\perp_{i} \equiv \min S_{i}$ ), while $z \in \tilde{R}_{i}\left(Q-T_{i}, t\right) \Rightarrow z-T_{i} \leq 0 .{ }^{38}$ It may be verified that $B_{i}(Q, t)$ is

\footnotetext{
${ }^{38}$ Here it is necessary to extend $\tilde{R}_{2}$ by defining $\tilde{R}_{2}(z)=\left\{\tilde{R}_{\imath}(0)\right\}$ when $z<0$, where we have here taken $\perp_{j}=0$ for all $j$ so that the least value $Q$ can assume is 0 . It is clear that with this extension $\bar{R}_{2}\left(Q-s_{i}\right)-\left\{s_{i}\right\}$ is $(s t i l l)$ descending and now always passes through 0 . Importantly, the extensions (one for each agent), do not introduce any new fixed points for $B=\sum_{j} B_{j}$ : Given $Q$, if $s_{i} \in \bar{R}_{i}\left(Q-s_{i}\right)$, then either $Q-s_{i} \geq 0$ or $Q<s_{i} \in \bar{R}_{2}\left(Q-s_{i}\right)$. But if $s_{i}>Q$ for just one $i$, we cannot have $\sum_{j} s_{j}=Q$.
} 


\section{References}

Acemoglu, D. (2007): "Equilibrium Bias of Technology", Econometrica 175, 1371-1410.

Ausubel, L.M. and R.J. Deneckere (1993): "A Generalized Theorem of the Maximum", Economic Theory 3, 99-107.

Bassett, L., J. Maybee and J. Quirk (1968): "Qualitative Economics and the Scope of the Correspondence Principle", Econometrica 36, 544-563.

Bergstrom, T., Blume, L. and H. Varian (1986): "On the Private Provision of Public Goods", Journal of Public Economics 29, 25-49.

Berman, A. and R.J. Plemmons, Nonnegative Matrices in the Mathematical Sciences, SIAM (Classics in Mathematics \# 9), 1994.

Cooper, R. and A. John (1988): "Coordinating Coordination Failures in Keynesian Models", Quarterly Journal of Economics 103, 441-463.

Corchón, L. (1994): "Comparative Statics for Aggregative Games. The Strong Concavity Case", Mathematical Social Sciences 28, 151-165.

Cornes, R. and R. Hartley (2005): "Asymmetric Contests with General Technologies", Economic Theory 26, 923-946.

Diamond, P. (1982): "Aggregate Demand Management in Search Equilibrium", Journal of Political Economy 90, 881-894.

Dixit, A. (1987): "Strategic Behavior in Contests", American Economic Review 77, 891-898.

Dubey, P., O. Haimanko, and A. Zapechelnyuk (2006): "Strategic Complements and Substitutes, and Potential games", Games and Economic Behavior 54, 77-94.

Gorman, W.M. (1968): "The Structure of Utility Functions", Review of Economic Studies 35, 367-390.

Hale, D., G. Lady, J. Maybee, and J. Quirk, Nonparametric Comparative Statics and Stability, Princeton University Press, 1999.

Hirshleifer, J. (1989): "Conflict and Rent-Seeking Success Functions: Ratio vs. Difference Models of Relative Success", Public Choice 63, 101-112.

Jensen, M.K. (2007): "Aggregative Games and Best-reply Potentials", Economic Theory, forthcoming.

Kukushkin, N.S. (1994): "A Fixed-point Theorem for Decreasing Mappings", Economics Letters $46,23-26$.

Loury, G.C. (1979): "Market Structure and Innovation", Quarterly Journal of Economics 93, $395-410$.

Mangasarian, O.L. (1965): "Pseudo-Convex Functions", SIAM Journal of Control 3, 281-290.

Milgrom, P. and J. Roberts (1990): "Rationalizability, Learning, and Equilibrium in Games with Strategic Complementarities", Econometrica 58, 1255-1277.

Milgrom, P. and J. Roberts (1994): "Comparing Equilibria", American Economic Review 84, 441-459. 
Milgrom, P. and C. Shannon (1994): "Monotone Comparative Statics", Econometrica 62, 157-180.

Milnor, J.W., Topology from the differentiable viewpoint, The University Press of Virginia, Charlottesville, 1965.

Nti, K.O. (1997): "Comparative Statics of Contests and Rent-Seeking Games", International Economic Review 38, 43-59.

Novshek, W. (1985): "On the Existence of Cournot Equilibrium", Review of Economic Studies $52,85-98$.

Possajennikov, A. (2003): "Evolutionary Foundation of Aggregative-taking Behavior", Economic Theory 21, 921-928.

Szidarovszky, F, and K. Okuguchi (1997): "On the Existence and Uniqueness of Pure Nash Equilibrium in Rent-Seeking Games", Games and Economic Behavior 18, 135-140.

Schipper, B. (2005): "Submodularity and the evolution of Walrasian behavior", International Journal of Game Theory 32, 471-477.

Selten, R., Preispolitik der Mehrproduktenunternehmung in der Statischen Theorie, Springer Verlag, Berlin, 1970.

Tarski, A. (1955): "A Lattice-theoretical Fixpoint Theorem and its Applications", Pacific Journal of Mathematics 5, 285-309.

Topkis, D.M. (1978): "Minimizing a Submodular Function on a Lattice", Operations Research, $26,305-321$.

Topkis, D.M. (1979): "Equilibrium Points in Nonzero-sum n-person Submodular Games", SIAM Journal of Control and Optimization 17, 773-787.

Topkis, D.M., Supermodularity and Complementarity, Princeton University Press, New Jersey, 1998.

Tullock, G. (1980): "Efficient rent-seeking". In: Buchanan, J.M., Tollison, R.D., Tullock, G. (Eds.), Toward a Theory of the Rent-Seeking Society. Texas A\&M Press, College Station, pp. $97-112$.

Vega-Redondo, F. (1997): "The Evolution of Walrasian Behavior", Econometrica 65, 375-384.

Villas-Boas, J.M. (1997): "Comparative Statics of Fixed Points", Journal of Economic Theory $73,183-198$.

Vind, K. and B. Grodal, Independence, Additivity, Uncertainty, Springer Verlag (Studies in Economic Theory Vol.14), Berlin, 2003.

Vives, X. (1990): "Nash Equilibrium with Strategic Complementarities", Journal of Mathematical Economics 19, 305-321.

Weinstein, J. and M. Yildiz (2008): "Sensitivity of Equilibrium Behavior to Higher-Order Beliefs in Nice Games", Mimeo, MIT Department of Economics. 